\title{
Borehole Resistivity Simulations of Oil-Water Transition Zones with a 1.5D Numerical Solver
}

\author{
M. Shahriari • D. Pardo
}

Received: date/ Accepted: date

\begin{abstract}
When simulating borehole resistivity measurements in a reservoir, it is common to consider an oilwater contact (OWC) planar interface. However, this consideration can lead to an unrealistic model since in the presence of capillary actions, the mix of two immiscible fluids (oil and water) often appears as an oil-water transition (OWT) zone. These transition zones may be significant in the vertical direction (20 meters or above), and in context of geosteering, an efficient method to simulate the OWT zone can maximize the production of an oil reservoir. Herein, we propose an efficient one and a half dimensional (1.5D) numerical solver to accurately simulate the OWT zone in an oil reservoir. Using this method, we can easily consider arbitrary resistivity distributions in the vertical direction, as it occurs in an OWT zone. Numerical results on synthetic examples demonstrate significant differences between the results recorded by a geosteering device when considering a realistic OWT zone vs an OWC sharp interface.
\end{abstract}

Keywords logging-while-drilling (LWD), resistivity measurements, deep azimuthal instrument, oil-water contact, reservoir geosteering, oil-water transition zone

\section{Shahriari}

Software Competence Center Hagenberg (SCCH), Hagenberg, Austria.

Softwarepark 21, 4232 Hagenberg im Mühlkreis, Austria.

E-mail: m.shahriari.sh@gmail.com.com

D. Pardo

University of the Basque Country (UPV/EHU), Leioa, Spain. Basque Center for Applied Mathematics, (BCAM), Bilbao, Spain.

Ikerbasque (Basque Foundation for Sciences), Bilbao, Spain. E-mail: dzubiaur@gmail.com

\section{Introduction}

Geophysical resistivity measurements are used to map the subsurface, explore hydrocarbon reservoirs, and maximize the production of the existing ones. We categorize existing resistivity measurements as: (1) on surface, such as those obtained using controlled source electromagnetic (CSEM) [1 5] and Magnetotellurics (MT) 6. 7]; and (2) borehole resistivity measurements, for example, those acquired using logging-while-drilling (LWD) devices 8 19. LWD devices are useful both for reservoir characterization [20 22 and geosteering purposes 23, 24, which is the act of adjusting the tool direction to travel through a specific zone.

Recently, deep and extra-deep azimuthal logging devices have been introduced as a new type of LWD instruments [14, 15]. In addition to map the subsurface, they help us to select the well trajectory properly in the hydrocarbon reservoir in order to increase the productivity of the well [14, 16 18. There exist several differences between conventional LWD devices and deep azimuthal devices, e.g., the number of transmitters and receivers, and the spacings between them, which are significantly larger in deep azimuthal configurations. Figure 1 displays a typical deep azimuthal device incorporating tilted coils and operating at several frequencies. The main advantage of deep and extra-deep resistivity instruments in comparison to conventional LWD devices is their significantly longer depth of investigation, which enables to perform geosteering without crossing a water-saturated rock [14, 15].

Geosteering requires solving multiple inverse problems in real time [8, 11. A full three-dimensional (3D) inversion is costly, which makes it often unsuitable for this application. An alternative computational solution consists of dividing our problem domain into a sequence 


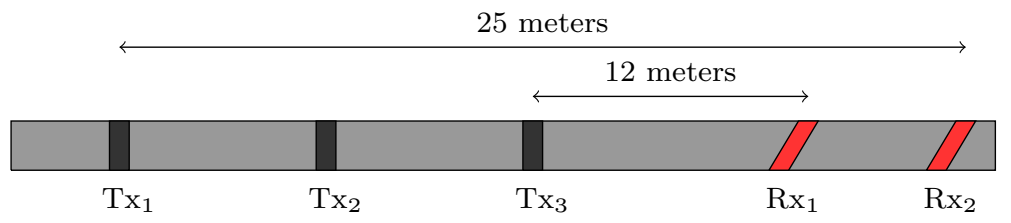

Fig. 1: A LWD deep azimuthal tool. $T_{1}, T_{2}, T_{3}$ are the transmitters, and $R_{1}$ and $R_{2}$ are two tilted receivers.

of $1 \mathrm{D}$ models in the proximity of the logging instrument, as shown in 8 . In the resulting $1 \mathrm{D}$ layered model, it is possible to reduce the dimensionality of the problem using a Hankel transform or a 2D Fourier transform along the directions over which the material properties are constant 9, 19, 25. By performing this dimensionality reduction, we obtain a system of ordinary differential equations (ODEs) that can be solved either: (a) analytically, by considering a piecewise constant resistivity profile, which leads to a so called semi-analytic method [25, 26], or (b) numerically [9, 19].

Despite the high efficiency of semi-analytic methods, they may sometimes lead to an unrealistic mapping of the reservoir since they have to consider piecewise constant resistivity profiles, and as a result, they often employ a sharp oil-water contact (OWC). However, in many realistic reservoir models, the OWC often appears as an oil-water saturated transition (OWT) zone with a variable resistivity profile (see Figure 2] 27, 30]. To model this with a $1.5 \mathrm{D}$ semi-analytical code, it is necessary to approximate the real model in the OWT zone using multiple constant-resistivity layers, which increases the computational cost, implementation, modeling error, and complexity of computing derivatives needed by gradient-based inversion methods.

In here, we propose a more natural approach to model the OWT zone, as well as other non-piecewiseconstant resistivity profiles that may appear in different formations. For that, we employ a 1.5D multi-scale finite element method (FEM) to solve the system of ODEs that arises after reducing the dimensionality of the problem utilizing a Hankel transform [19]. The proposed method can model arbitrary $1 \mathrm{D}$ resistivity distributions of the subsurface (as it occurs on an OWT zone), and not just a piecewise constant distribution as in the case of semi-analytic methods. Moreover, using a multi-scale method, we reach the high efficiency required for the application while avoiding unnecessary approximations of the resistivity model in the OWT zone. We illustrate numerically the performance of the method via two synthetic examples in which the saturation profiles are obtained from the experiment described in 30], and the corresponding resistivity profiles are estimated using Archie's (experimental) law 31.
We illustrate our proposed approach with a practical geosteering example.

We organize the rest of the document as follows: Section 2 describes Maxwell's equations, which govern the physics of our borehole resistivity problems. Section 3 derives the corresponding variational formulation. Section 4 provides a brief description of our proposed 1.5D solver. In Section 5, we employ Archie's law to calculate the resistivity of a fluid saturated rock, we provide examples of OWT zones, and two synthetic model problems. Section 6 verifies the efficiency of our numerical solver by modeling the synthetic examples introduced in Section 5. Section 7 illustrates the application of our approach to a realistic geosteering example. Section 8 is dedicated to the conclusions.

\section{Governing equation}

We consider $\boldsymbol{E}$ and $\boldsymbol{H}$ to be the complex-valued electric and magnetic fields, respectively. We denote $\boldsymbol{\sigma}(x, y, z)$ to be the real-valued conductivity tensor with positive determinant. Let $\mathbf{M}$ be a magnetic source flux density. Then, Maxwell's equations governs the propagation of the EM fields in 3D space as follows [32]:

$$
\begin{aligned}
& \nabla \times \boldsymbol{H}=(\boldsymbol{\sigma}-i \omega \varepsilon) \boldsymbol{E}, \\
& \nabla \times \boldsymbol{E}=i \omega \boldsymbol{\mu} \boldsymbol{H}+i \omega \boldsymbol{\mu} \mathbf{M},
\end{aligned}
$$

where $\omega=2 \pi f$ is the angular frequency, where $f>0$ is the frequency of the transmitter, $\boldsymbol{\varepsilon}$ and $\boldsymbol{\mu}$ are the permittivity and magnetic permeability tensors of the media, respectively, and $i$ is the imaginary unit, i.e., $i^{2}=-1$. The problem is defined over the entire space $\Omega=\mathbb{R}^{3}$.

We now pre-multiply (1) by $\tilde{\boldsymbol{\sigma}}^{-1}=(\boldsymbol{\sigma}-i \omega \varepsilon)^{-1}$, and we apply the curl operator. Then, we substitute (2) into the result and we obtain the following reduced wave equation in terms of the magnetic field:

$$
\nabla \times \tilde{\boldsymbol{\sigma}}^{-1} \nabla \times \boldsymbol{H}-i \omega \boldsymbol{\mu} \boldsymbol{H}=i \omega \boldsymbol{\mu} \mathbf{M}
$$

\section{Variational formulation}

We consider $\boldsymbol{F}$ to be an arbitrary vector-valued test function and $\boldsymbol{F}^{*}$ its conjugate transpose. We pre-multiply 


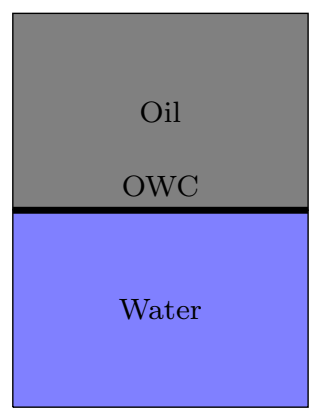

(a) OWC surface

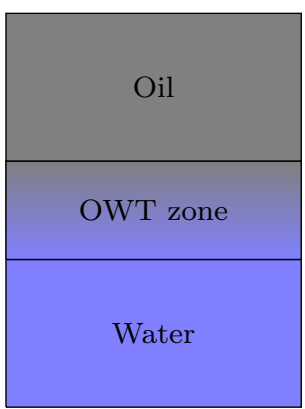

(b) OWT zone

Fig. 2: Oil-water contact surface and transition zone.

(3) by $\boldsymbol{F}^{*}$, and we integrate over the entire domain to arrive at:

$$
\begin{aligned}
\int_{\Omega} \boldsymbol{F}^{*}\left(\nabla \times \tilde{\boldsymbol{\sigma}}^{-1} \nabla \times \boldsymbol{H}\right) d \Omega & -i \omega \int_{\Omega} \boldsymbol{F}^{*} \boldsymbol{\mu} \boldsymbol{H} d \Omega \\
& =i \omega \int_{\Omega} \boldsymbol{F}^{*} \boldsymbol{\mu} \mathbf{M} d \Omega
\end{aligned}
$$

Using integration by parts, and considering that the magnetic field decreases exponentially while moving away from the transmitter, we obtain the following variational formulation:

$$
\begin{array}{r}
\int_{\Omega}(\nabla \times \boldsymbol{F})^{*}\left(\tilde{\boldsymbol{\sigma}}^{-1} \nabla \times \boldsymbol{H}\right) d \Omega \\
-i \omega \int_{\Omega} \boldsymbol{F}^{*} \boldsymbol{\mu} \boldsymbol{H} d \Omega \\
=i \omega \int_{\Omega} \boldsymbol{F}^{*} \boldsymbol{\mu} \mathbf{M} d \Omega .
\end{array}
$$

In order to guarantee integrability of all terms appearing in (5), we select $\boldsymbol{F}, \boldsymbol{H} \in H(\mathbf{c u r l} ; \Omega)$, where:

$H(\operatorname{curl} ; \Omega)=\left\{\boldsymbol{F} \in\left(L^{2}(\Omega)\right)^{3}: \nabla \times \boldsymbol{F} \in\left(L^{2}(\Omega)\right)^{3}\right\}$.

\section{1.5D variational formulation}

In [19, we propose a $1.5 \mathrm{D}$ variational formulation to obtain the magnetic field in a 1D layered media. Herein, we summarize those derivations for this work to be selfcontained.

We consider a 1D Transversely Isotropic (TI) layered media. Thus, the conductivity varies only along $z$ direction, and we restrict to conductivity tensors of the type:

$\boldsymbol{\sigma}(z)=\left(\begin{array}{ccc}\sigma_{h}(z) & 0 & 0 \\ 0 & \sigma_{h}(z) & 0 \\ 0 & 0 & \sigma_{v}(z)\end{array}\right)$

where $\sigma_{h}>0$ and $\sigma_{v}>0$ are the horizontal and vertical conductivity of the media, respectively. Analogously, we consider $\varepsilon(z)$ and $\boldsymbol{\mu}(z)$ to be 1D transversely isotropic.
We consider $\boldsymbol{H}=\left(H_{x}, H_{y}, H_{z}\right)$ to be the magnetic field in the Cartesian system of coordinates. Since the material properties vary only along $z$ direction, it is convenient to use a $2 \mathrm{D}$ Fourier transform along $x y$ plane as follows:

$$
\begin{aligned}
\boldsymbol{H}(x, y, z):=\frac{1}{4 \pi^{2}} \int_{-\infty}^{+\infty} \int_{-\infty}^{+\infty} \widehat{\boldsymbol{H}}\left(k_{x}, k_{y}, z\right) \\
\cdot e^{i\left(k_{x}, k_{y}\right) \cdot(x, y)} d k_{x} d k_{y}
\end{aligned}
$$

where $\widehat{\boldsymbol{H}}$ is the Fourier transform of the magnetic field, and $k_{x}$ and $k_{y}$ are the Fourier modes.

Using the following transformations:

$x=\rho \cdot \cos \phi, y=\rho \cdot \sin \phi$,

$k_{x}=\xi \cdot \cos \theta, k_{y}=\xi \cdot \sin \theta$,

we change our system of coordinates from Cartesian to cylindrical, and we obtain:

$$
\begin{aligned}
\boldsymbol{H}(\rho, \phi, z)=\frac{1}{4 \pi^{2}} \int_{0}^{+\infty} & \int_{0}^{2 \pi} \widehat{\boldsymbol{H}}(\xi, \theta, z) \\
& \cdot e^{i \xi \rho(\cos \theta \cos \phi+\sin \theta \sin \phi)} d \theta \xi d \xi .
\end{aligned}
$$

Using the trigonometric identity $\cos (\phi-\theta)=\cos \theta \cos \phi+$ $\sin \theta \sin \phi$, we obtain:

$$
\begin{aligned}
\boldsymbol{H}(\rho, \phi, z)=\frac{1}{4 \pi^{2}} \int_{0}^{+\infty} \int_{0}^{2 \pi} \widehat{\boldsymbol{H}}(\xi, \theta, z) \\
\cdot e^{i \xi \rho \cos (\phi-\theta)} d \theta \xi d \xi .
\end{aligned}
$$

We have the following relation between exponentials and Bessel functions:

$e^{i \xi \rho \cos (\phi-\theta)}=\sum_{k=-\infty}^{\infty} i^{k} J_{k}(\xi \rho) e^{-i k(\phi-\theta)}$. 
Substituting (11) into (10), we have:

$\boldsymbol{H}(\rho, \phi, z)=\frac{1}{2 \pi} \sum_{k=-\infty}^{\infty} \int_{0}^{+\infty} \boldsymbol{H}^{k}(\xi, z) J_{k}(\xi \rho) e^{-i k \phi} \xi d \xi$,

where

$\boldsymbol{H}^{k}(\xi, z)=\frac{1}{2 \pi} \int_{0}^{2 \pi} \widehat{\boldsymbol{H}}(\xi, \theta, z) i^{k} e^{i k \theta} d \theta$.

Moreover, the cylindrical components of the magnetic field are as follows:

$$
\begin{aligned}
H_{\rho}^{k} & =\cos \phi \cdot H_{x}^{k}+\sin \phi \cdot H_{y}^{k} \\
& =e^{i \phi} H_{+}^{k}+e^{-i \phi} H_{-}^{k}, \\
H_{\phi}^{k} & =-\sin \phi \cdot H_{x}^{k}+\cos \phi \cdot H_{y}^{k} \\
& =i\left(e^{i \phi} H_{+}^{k}-e^{-i \phi} H_{-}^{k}\right),
\end{aligned}
$$

where

$$
\begin{aligned}
& H_{+}^{k}=\frac{H_{x}^{k}-i H_{y}^{k}}{2}, \\
& H_{-}^{k}=\frac{H_{x}^{k}+i H_{y}^{k}}{2} .
\end{aligned}
$$

By substituting (14) into (12), and using (15), the Hankel representation of the magnetic field becomes:

$$
\begin{array}{r}
H_{\rho}(\rho, \phi, z)=\frac{1}{2 \pi} \sum_{k=-\infty}^{+\infty} e^{-i k \phi} \int_{0}^{+\infty}\left(H_{+}^{k}(\xi, z) J_{k+1}(\xi \rho)\right. \\
\left.+H_{-}^{k}(\xi, z) J_{k-1}(\xi \rho)\right) \xi d \xi,
\end{array}
$$

$H_{\phi}(\rho, \phi, z)=\frac{i}{2 \pi} \sum_{k=-\infty}^{+\infty} e^{-i k \phi} \int_{0}^{+\infty}\left(H_{+}^{k}(\xi, z) J_{k+1}(\xi \rho)\right.$ $\left.-H_{-}^{k}(\xi, z) J_{k-1}(\xi \rho)\right) \xi d \xi$,

$H_{z}(\rho, \phi, z)=\frac{1}{2 \pi} \sum_{k=-\infty}^{+\infty} e^{-i k \phi} \int_{0}^{+\infty} H_{z}^{k}(\xi, z) J_{k}(\xi \rho) \xi d \xi$.

For a Hankel mode $\xi_{q}>0$ and an exponential order $t$, we select the following mono-modal test functions:

$$
\begin{array}{r}
\boldsymbol{F}^{q, t}(\rho, \phi, z)=F_{\rho}^{q, t}(\rho, \phi, z) \hat{\boldsymbol{\rho}} \\
+F_{\phi}^{q, t}(\rho, \phi, z) \hat{\boldsymbol{\phi}} \\
+F_{z}^{q, t}(\rho, \phi, z) \hat{\mathbf{z}},
\end{array}
$$

where:

$$
\begin{array}{rr}
F_{\rho}^{q, t}(\rho, \phi, z)= & e^{-i t \phi}\left(F_{+}^{t}\left(\xi_{q}, z\right) J_{t+1}\left(\xi_{q} \rho\right)\right. \\
& \left.+F_{-}^{t}\left(\xi_{q}, z\right) J_{t-1}\left(\xi_{q} \rho\right)\right), \\
F_{\phi}^{q, t}(\rho, \phi, z)= & i e^{-i t \phi}\left(F_{+}^{t}\left(\xi_{q}, z\right) J_{t+1}\left(\xi_{q} \rho\right)\right. \\
& \left.-F_{-}^{t}\left(\xi_{q}, z\right) J_{t-1}\left(\xi_{q} \rho\right)\right), \\
F_{z}^{q, t}(\rho, \phi, z)= & e^{-i t \phi} F_{z}^{t}\left(\xi_{q}, z\right) J_{t}\left(\xi_{q} \rho\right) .
\end{array}
$$

In order to exploit the orthogonality of Bessel functions, we define the following rotation (unitary) matrix:

$Q=\frac{1}{\sqrt{2}}\left(\begin{array}{ccc}1 & i & 0 \\ i & 1 & 0 \\ 0 & 0 & \sqrt{2}\end{array}\right)$,

and we replace the variational formulation (5) by the following equivalent one:

$$
\begin{aligned}
& \int_{\Omega}(Q \nabla \times \boldsymbol{F})^{*}\left(Q \tilde{\boldsymbol{\sigma}}^{-1} \nabla \times \boldsymbol{H}\right) d \Omega \\
& -i \omega \int_{\Omega}(Q \boldsymbol{F})^{*}(Q \boldsymbol{\mu} \boldsymbol{H}) d \Omega=i \omega \int_{\Omega} \boldsymbol{F}^{*} \boldsymbol{\mu} \mathbf{M} d \Omega .
\end{aligned}
$$

Notice that Equations (20) and (5) are equivalent because $Q$ is a unitary matrix, so $Q * Q=I$. For an arbitrary function $\mathbf{g}(\xi, z)=\left(g_{x}(\xi, z), g_{y}(\xi, z), g_{z}(\xi, z)\right)$ in the spectral domain, we denote:

$$
\begin{aligned}
& g_{+}(\xi, z):=\frac{g_{x}(\xi, z)-i g_{y}(\xi, z)}{2}, \\
& g_{-}(\xi, z):=\frac{g_{x}(\xi, z)+i g_{y}(\xi, z)}{2},
\end{aligned}
$$

and

$$
\begin{aligned}
& \Pi_{+}^{\xi}(\mathbf{g}(\xi, z)):=\frac{\partial g_{+}(\xi, z)}{\partial z}+\frac{\xi}{2} g_{z}(\xi, z), \\
& \Pi_{-}^{\xi}(\mathbf{g}(\xi, z)):=\frac{\partial g_{-}(\xi, z)}{\partial z}-\frac{\xi}{2} g_{z}(\xi, z), \\
& \Pi_{z}^{\xi}(\mathbf{g}(\xi, z)):=\xi\left(g_{-}(\xi, z)+g_{+}(\xi, z)\right) .
\end{aligned}
$$

By substituting (16) and (18) into the variational formulation (20), and using (21) and 222, and the orthogonality of Bessel functions, for each Hankel mode $\xi_{q}$, we obtain the following variational formulation:

$b\left(\boldsymbol{F}^{q, m}, \boldsymbol{H}\right)=b\left(\mathbf{F}^{m}, \mathbf{H}^{m}\right)=b_{1}\left(\mathbf{F}^{m}, \mathbf{H}^{m}\right)-b_{2}\left(\mathbf{F}^{m}, \mathbf{H}^{m}\right)$,

where

$$
\begin{aligned}
b_{1}\left(\mathbf{F}^{m}, \mathbf{H}^{m}\right) & =2\left\langle\Pi_{-}^{\xi_{q}}\left(\mathbf{F}^{m}\right), \tilde{\sigma}_{h}^{-1} \Pi_{-}^{\xi_{q}}\left(\mathbf{H}^{m}\right)\right\rangle_{L^{2}} \\
& +2\left\langle\Pi_{+}^{\xi_{q}}\left(\mathbf{F}^{m}\right), \tilde{\sigma}_{h}^{-1} \Pi_{+}^{\xi_{q}}\left(\mathbf{H}^{m}\right)\right\rangle_{L^{2}} \\
& +\left\langle\Pi_{z}^{\xi_{q}}\left(\mathbf{F}^{m}\right), \tilde{\sigma}_{v}^{-1} \Pi_{z}^{\xi_{q}}\left(\mathbf{H}^{m}\right)\right\rangle_{L^{2}}, \\
b_{2}\left(\mathbf{F}^{m}, \mathbf{H}^{m}\right) & =i \omega \mu_{0}\left(2\left\langle F_{-}^{m}, H_{-}^{m}\right\rangle_{L^{2}}+2\left\langle F_{+}^{m}, H_{+}^{m}\right\rangle_{L^{2}}\right. \\
& \left.+\left\langle F_{z}^{m}, H_{z}^{m}\right\rangle_{L^{2}}\right),
\end{aligned}
$$

and $\langle f, g\rangle_{L^{2}}=\int_{z} \bar{f} g d z$. For the above formulation to be integrable, we request $\mathbf{H}^{m}, \mathbf{F}^{m} \in V(\mathbb{R})$, where $V(\mathbb{R})=$ $H^{1}(\mathbb{R}) \times H^{1}(\mathbb{R}) \times L^{2}(\mathbb{R})$, and

$H^{1}(\mathbb{R})=\left\{v \in L^{2}(\mathbb{R}): \frac{\partial v}{\partial z} \in L^{2}(\mathbb{R})\right\}$. 
For each Hankel mode $\xi_{q}$, we solve the aforementioned variational formulation for the following right-hand sides:

$$
l\left(\mathbf{F}^{m}\right)= \begin{cases}i \omega \mu_{h}\left(z_{T x}\right)\left(F_{+}^{-1}\left(\xi_{q}, z_{T x}\right)\right)^{*} & m=-1, \\ i \omega \mu_{v}\left(z_{T x}\right)\left(F_{z}^{0}\left(\xi_{q}, z_{T x}\right)\right)^{*} & m=0, \\ i \omega \mu_{h}\left(z_{T x}\right)\left(F_{-}^{1}\left(\xi_{q}, z_{T x}\right)\right)^{*} & m=1,\end{cases}
$$

where $z_{T x}$ is the location of the transmitter along $z$ direction. We define:

$$
\begin{aligned}
H_{\rho}^{m}(\rho, \phi, z)= & \frac{1}{2 \pi} e^{-i m \phi} \int_{0}^{+\infty}\left(H_{+}^{m}(\xi, z) J_{m+1}(\xi \rho)\right. \\
& \left.+H_{-}^{m}(\xi, z) J_{m-1}(\xi \rho)\right) \xi d \xi \\
H_{\phi}^{m}(\rho, \phi, z)= & \frac{i}{2 \pi} e^{-i m \phi} \int_{0}^{+\infty}\left(H_{+}^{m}(\xi, z) J_{m+1}(\xi \rho)\right. \\
& \left.-H_{-}^{m}(\xi, z) J_{m-1}(\xi \rho)\right) \xi d \xi \\
H_{z}^{m}(\rho, \phi, z)= & \frac{1}{2 \pi} e^{-i m \phi} \int_{0}^{+\infty} H_{z}^{m}(\xi, z) J_{m}(\xi \rho) \xi d \xi
\end{aligned}
$$

Then, for each transmitter orientation, the magnetic field is:

$\boldsymbol{H}= \begin{cases}\boldsymbol{H}^{1}+\boldsymbol{H}^{-1} & x \text {-oriented } \\ i \boldsymbol{H}^{1}-i \boldsymbol{H}^{-1} & y \text {-oriented } \\ \boldsymbol{H}^{0} & z \text {-oriented }\end{cases}$

The derived variational formulation allows the material properties to vary along $z$ direction arbitrarily and, in particular, we can accurately model an OWT zone. Moreover, to achieve high efficiency, we use the multi-scale method proposed in [19].

\section{Resistivity profiles and synthetic subsurface models and logging trajectories}

The OWT zone is the area of the formation that separates the oil-saturated reservoir from the water-saturated rock (see Figure 2b). OWT zones where the water saturation $\left(S_{w}\right)$ is below one appear because of a loss of buoyancy pressure in the hydrocarbon phase.

Determining the water saturation profile in the transition zone in a porous media to predict the recoverable oil saturated in the rock is a demanding task in petrophysics (see, e.g., [27 29]). However, sometimes, a saturation profile may be available from previously recorded data. For example, in 30, the authors utilized electrical wireline logs and capillary pressure relations to obtain the water saturation in the Wellington West field. In here, we use the data provided by 30] to produce two realistic examples of OWT zones (see Table 1).

\begin{tabular}{|r|c|c|}
\hline$S_{w}$ (fraction) & Model 1 (meters) & Model 2 (meters) \\
\hline 0.05 & 0.0 & - \\
\hline 0.1 & 14.02 & - \\
\hline 0.15 & 18.59 & 0 \\
\hline 0.18 & - & 4.57 \\
\hline 0.2 & 21.33 & 9.14 \\
\hline 0.25 & 23.16 & 12.19 \\
\hline 0.3 & 24.38 & 14.63 \\
\hline 0.35 & 24.84 & 16.15 \\
\hline 0.4 & 25.29 & 17.83 \\
\hline 0.45 & 25.75 & 19.05 \\
\hline 0.5 & 26.21 & 20.26 \\
\hline 0.55 & 26.67 & 21.33 \\
\hline 0.6 & 27.12 & 21.94 \\
\hline 0.65 & 27.27 & 22.55 \\
\hline 0.7 & 27.43 & 23.16 \\
\hline 0.75 & 27.55 & 23.46 \\
\hline 0.8 & 27.67 & 23.77 \\
\hline 0.85 & 27.79 & 23.92 \\
\hline 0.9 & 27.91 & 24.07 \\
\hline 0.95 & 28.04 & 24.23 \\
\hline 1 & 28.16 & \\
\hline & &
\end{tabular}

Table 1: Water saturation in two transition zones.

In order to compute the resistivity of a fluid saturated rock, we employ Archie's law, given by:

$\rho_{o}=a \phi^{-m} S_{w}^{-n} \rho_{w}$,

where $\rho_{o}$ is the resistivity of the oil-saturated rock, $\rho_{w}$ is the brine resistivity (in our case, we select $\rho_{w}=$ $0.13 \Omega \cdot m), a$ is the tortuosity factor, $\phi$ denotes the porosity, and $m$ and $n$ are the cementation and saturation exponents of the rock, respectively. In this work, we consider $m=n=2$ and $a=1$. Moreover, we assume a homogeneous rock porosity equal to $20 \%$ and $25 \%$ for our first and second models, respectively. Using $(29)$ and the resistivity values of Table 1, we obtain a set of resistivity values which are extended to the entire transition zone by using a cubic spline interpolation composed of three subintervals (see Figure 3 .

In this work, we approximate our formation as a sequence of three 1D models, which are described in Figures 4 and 5. Synthetic example 1 (Figure 4) considers the OWT zone described in Model 1 (Figure 3a), while synthetic example 2 (Figure 5 ) employs the OWT zone described in Model 2 (Figure 3b).

\begin{tabular}{|l|c|c|c|c|}
\hline Example & & & OWT zone & \\
\hline Example 1 $(\Omega \cdot m)$ & 10.00 & 1300 & $\rho_{s p, 1}$ & 3.25 \\
\hline Example 2 $(\Omega \cdot m)$ & 10.00 & 64.19 & $\rho_{s p, 2}$ & 2.08 \\
\hline
\end{tabular}

Table 2: Resistivity of different layers for our two synthetic examples.

In these examples, we assume for simplicity $\boldsymbol{\mu}=$ $\mu_{0} \mathbf{I}_{3}$ and $\boldsymbol{\varepsilon}=\varepsilon_{0} \mathbf{I}_{3}$ (where $\mathbf{I}_{3}$ is the $3 \mathrm{D}$ identity matrix) to be globally constant. $\varepsilon_{0}$ is set to $8.85 \times 10^{-12}(\mathrm{~F} / \mathrm{m})$, which corresponds to the free-space permittivity, while $\mu_{0}$ is set to $4 \pi \times 10^{-7}(H / m)$, i.e., the magnetic permeability constant. 


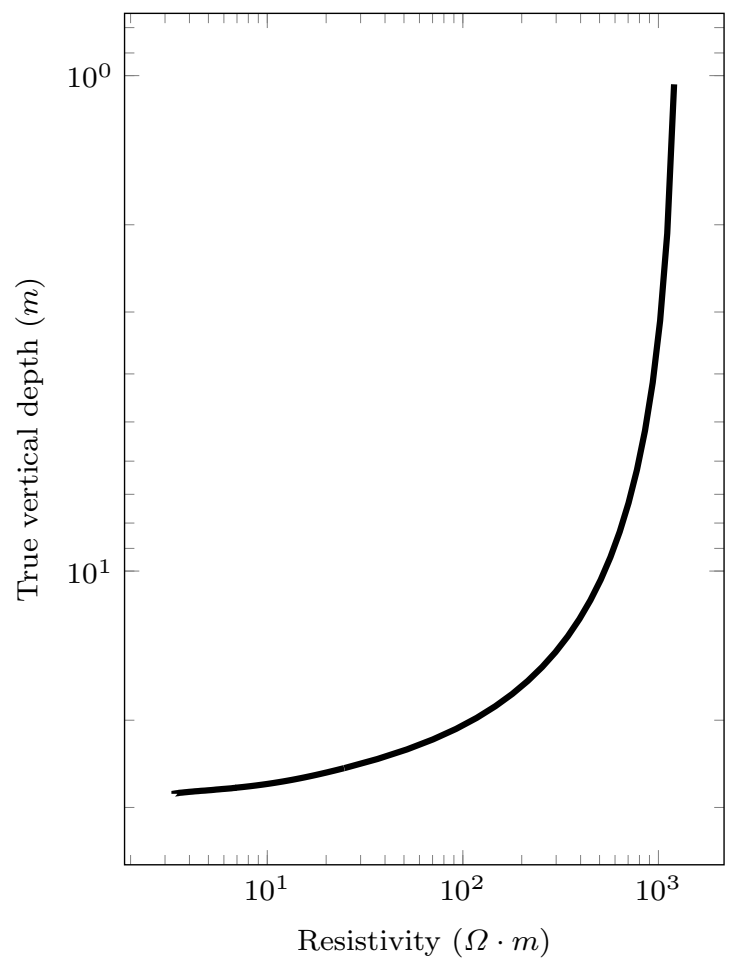

(a) Model $1\left(\rho_{s p, 1}\right)$

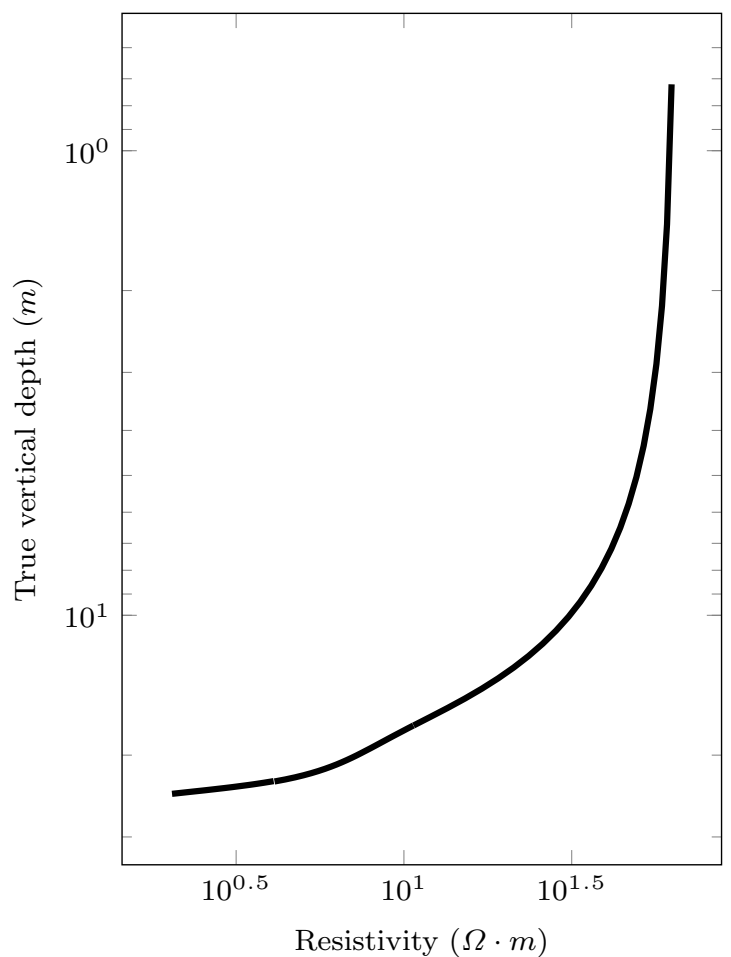

(b) Model $2\left(\rho_{s p, 2}\right)$

Fig. 3: Resistivity profile for two OWT zones.

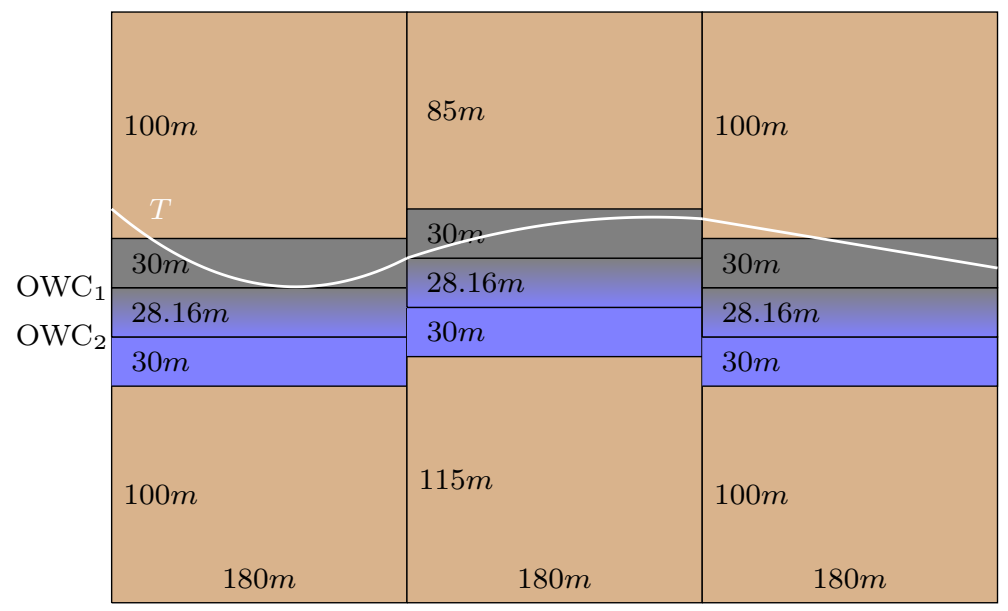

Fig. 4: Example 1. $T$ describes the logging trajectory.

\section{Numerical examples}

6.1 Logging device and measurements

We consider an extra-deep LWD instrument with short and long spacings, as described in Figure 6. The logging instrument operates at two frequencies, namely, $10 \mathrm{kHz}$ (short spacing) and $2 \mathrm{kHz}$ (long spacing).
We denote the nine couplings of the magnetic field as follows:

$$
\boldsymbol{H}=\left[\begin{array}{lll}
H_{x x} & H_{x y} & H_{x z} \\
H_{y x} & H_{y y} & H_{y z} \\
H_{z x} & H_{z y} & H_{z z}
\end{array}\right],
$$

where the first and the second subscripts refer to the direction of the transmitter and the receiver magnetic dipole, respectively. 


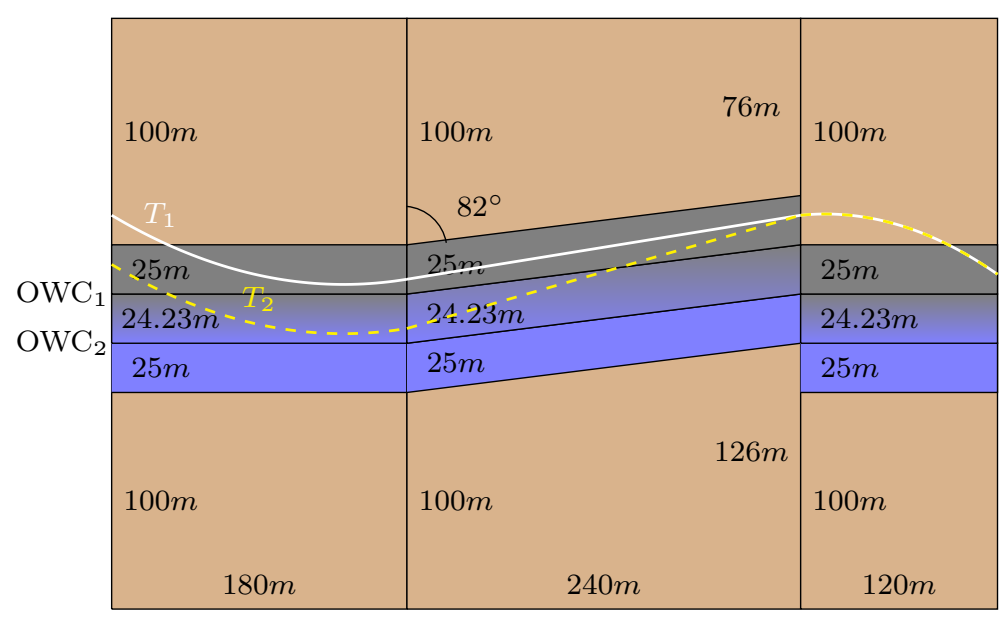

Fig. 5: Example 2. $T_{1}$ and $T_{2}$ describe two logging trajectories.

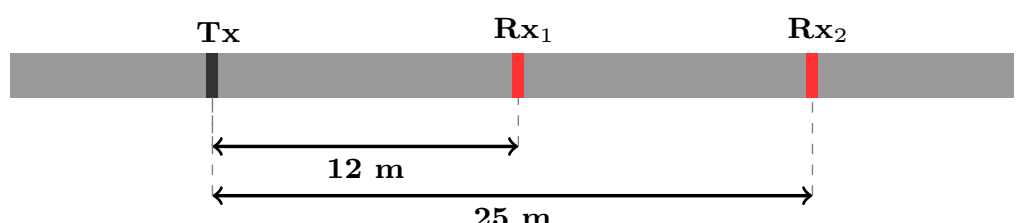

Fig. 6: Logging instrument. $\mathrm{Tx}$ is the induction transmitter, and $\mathrm{Rx}_{1}$ and $\mathrm{Rx}_{2}$ are the receivers. The instrument operates at two frequencies: $2 \mathrm{kHz}$ for the long spacing, and $10 \mathrm{kHz}$ for the short one.

In LWD resistivity measurements, it is common to consider attenuations and phase differences of the $z z$ coupling 33, 34. In our case, we consider an extradeep azimuthal instrument equipped with one receiver. Therefore, we define:

$$
\begin{aligned}
\ln \left(H_{z z}\right)= & \underbrace{} \underbrace{\ln \left(\left|H_{z z}\right|\right)} \\
+ & i \underbrace{\log (e)=\text { attenuation }(d B)} \\
& \times \frac{180}{\pi}=\text { phase difference (degrees) }
\end{aligned}
$$

Moreover, we consider directional measurements based on the apparent conductivity [23]. Following (30), we compute apparent conductivity $\boldsymbol{\sigma}_{p}$ as:

$$
\boldsymbol{\sigma}_{p}^{\prime}=\left[\begin{array}{lll}
\sigma_{p, x x} & \sigma_{p, x y} & \sigma_{p, x z} \\
\sigma_{p, y x} & \sigma_{p, y y} & \sigma_{p, y z} \\
\sigma_{p, z x} & \sigma_{p, z y} & \sigma_{p, z z}
\end{array}\right]^{\prime}=\mathbf{K} \cdot \boldsymbol{H}^{\prime}
$$

where the prime symbol denotes the adjoint of the matrix, and we have:

$$
\mathbf{K}=\left[\begin{array}{ccc}
i \frac{8 \pi}{\omega \mu_{0}} & i \frac{8 \pi}{\omega \mu_{0}} & i \frac{16 \pi}{\omega \mu_{0}} \\
i \frac{8 \pi}{\omega \mu_{0}} & i \frac{8 \pi}{\omega \mu_{0}} & i \frac{16 \pi}{\omega \mu_{0}} \\
i \frac{16 \pi}{\omega \mu_{0}} & i \frac{16 \pi}{\omega \mu_{0}} & i \frac{4 \pi}{\omega \mu_{0}}
\end{array}\right] .
$$

Using apparent conductivity (32), we define the symmetrized directional measurement as:

$\sigma_{d}=\frac{\sigma_{p, x z}-\sigma_{p, z x}}{2}$

The aforementioned directional measurement has high sensitivity to the bed boundaries [17, 20, 23. Another directional measurement which is highly sensitive to 
bed boundaries is the geosignal [35, which is defined as:

$$
\begin{aligned}
& g=\ln \left(\frac{H_{z z}+H_{z x}}{H_{z z}-H_{z x}}\right) \\
& =\underbrace{\ln \left(\frac{\left|H_{z z}+H_{z x}\right|}{\left|H_{z z}-H_{z x}\right|}\right)}_{\times 20 \log (e)=\text { attenuation }(d B)} \\
& +i \underbrace{\left(p h\left(H_{z z}+H_{z x}\right)-p h\left(H_{z z}-H_{z x}\right)\right)}_{\times \frac{180}{\pi}=\text { phase difference (degrees) }} .
\end{aligned}
$$

To illustrate that OWC models can lead to unrealistic simulations, we compare the simulated results corresponding to two OWC models (OWC1 and OWC2, see Figures 4 and 5 with the true OWT zone model.

\subsection{Validation}

To validate our numerical method, we compare our numerical solution to the semi-analytic one. We consider the $z z$ coupling of the magnetic field for the first example containing $\mathrm{OWC}_{2}$ using short spacing. Figure 7 shows the accuracy of our method (see 19] for further details about the method and its efficiency).

\subsection{Results on OWT zone vs OWC models}

Figure 8 shows the attenuation of the $z z$ coupling of the magnetic field for the first example using extra-deep short and long spacings. This figure shows significant differences between the $\mathrm{OWT}$ zone and $\mathrm{OWC}_{1}$ model results. The $O W C_{1}$ model leads to a large error in our simulated measurements, which will severely affect the inversion process. However, the aforementioned figures show a small difference between the OWT zone and $\mathrm{OWC}_{2}$ model solutions.

Figures 9 and 10 illustrate the apparent conductivity and geosignal, respectively, using extra-deep short and long spacings for the first example. As observed in the figures, the aforementioned directional measurements are highly sensitive to the position of a bed boundary. Each peak indicates the position of a bed boundary. The peak direction identifies the tool motion from a more resistive layer to a less resistive one (convex) or vice versa (concave). This information can be used for geosteering purposes to navigate the instrument inside the reservoir. Similar to the $z z$ coupling, there is a large difference between OWT zone and $\mathrm{OWC}_{1}$ model solutions, which can lead to a considerable inversion error.
Again, $\mathrm{OWC}_{2}$ model solution seems to provide similar logs to those obtained on the OWT zone scenario. However, these results may lead us to enter into a rock with a significant water saturation, thus, lowering the productivity. In summary, considering $\mathrm{OWC}_{2}$ instead of OWT zone puts us in the danger of entering the partially water saturated rock. Moreover, directional measurements help us to keep the well trajectory within the reservoir and modify its direction when necessary.

For the second example shown in Figure 5 , we consider two different trajectories. The first trajectory $T_{1}$ is entirely above the OWT zone. The results for $T_{1}$ verify, once again, the conclusions we obtained for the first example (see Figure 11). Moreover, to investigate the difference between $\mathrm{OWT}$ zone and $\mathrm{OWC}_{2}$ models, we consider a second trajectory $T_{2}$ which enters the OWT zone (see Figure 5. Figure 12 shows the attenuations and phase differences of the $z z$ coupling using the short spacing. In the aforementioned figure, a noticeable difference exists between the $\mathrm{OWT}$ and $\mathrm{OWC}_{2}$ models in the part of the trajectory that is inside the OWT zone. These differences may lead to important errors in our inverted values. Figures 13 and 14 show the directional measurements using short and long spacings. They describe a considerable difference between the OWT zone and $\mathrm{OWC}_{2}$ model solutions when the trajectory enters the OWT zone. Again, this difference may lead to a significant inversion error. Moreover, by considering $\mathrm{OWC}_{2}$, we are unable of accurately estimate the exact bed boundary positions. The aforementioned feebleness puts us in danger of entering the water saturated rock and creating attics in the reservoir. Consequently, the production of the reservoir may decrease if OWC models are considered.

\section{A practical geosteering example}

Figure 15 shows a model problem extracted from a field example with hydrocarbon-bearing layers. It is modeled as a sequence of $1 \mathrm{D}$ layered formations. Each 1D layered formation contains an oil-bearing reservoir, an OWT zone, and a water-saturated rock. The OWT zone is analogous to the one of the second example described in Figure 5. We consider two different synthetic trajectories, as illustrated in Figure 15. Figure 16 shows the evolution of the absolute value of the geosignal's attenuation as well as attenuation of the co-axial component using the short spacing for trajectory $T_{1}$. Figure 16 shows that the geosignal increases as we approach a more conductive layer, which suggests us to modify the well trajectory in order to keep it within the reservoir zone. Warned by this increase in the geosignal, the trajectory is effectively modified, maintaining the 


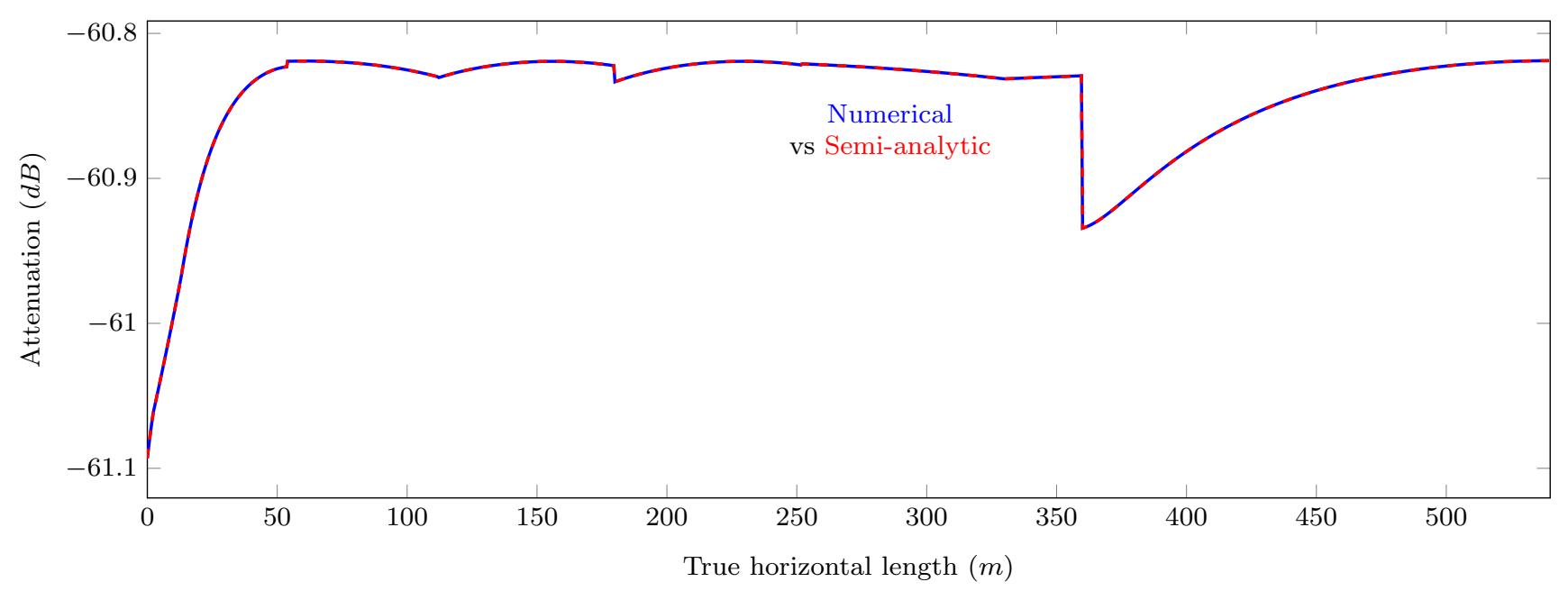

(a) Attenuation

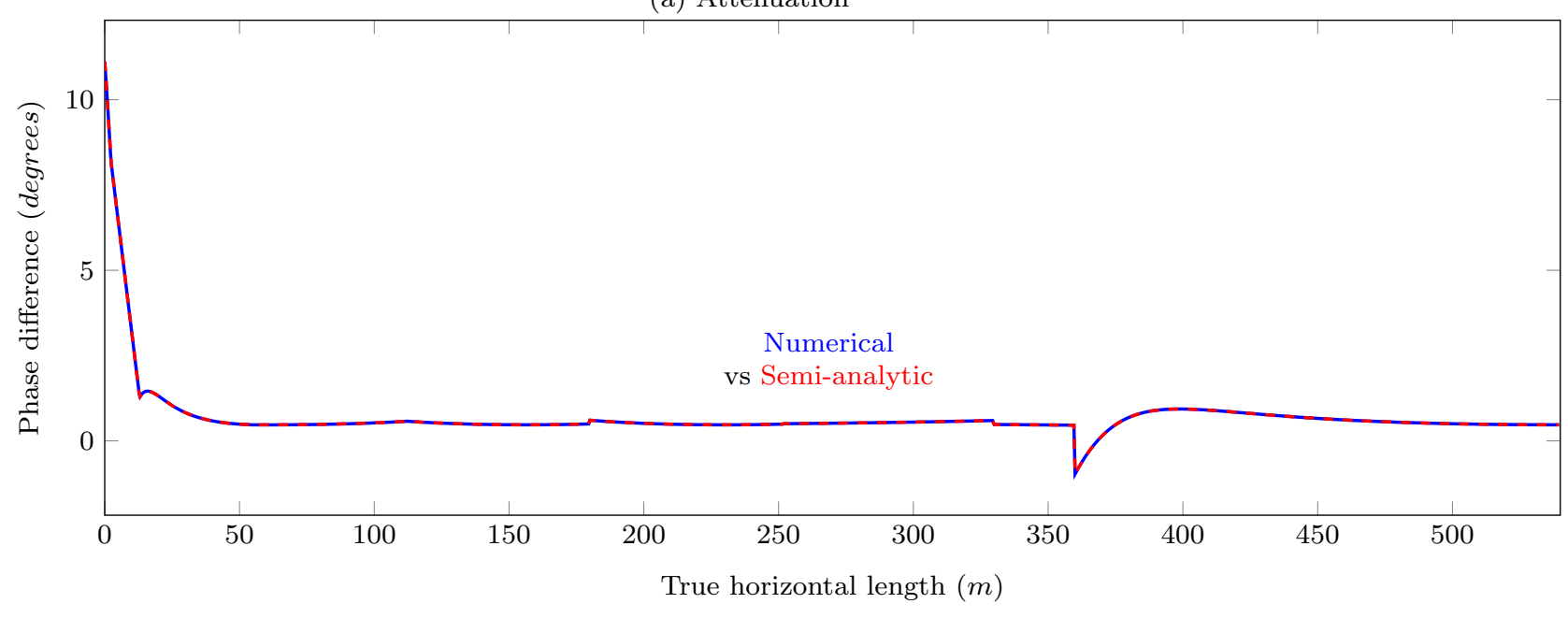

(b) Phase difference

Fig. 7: Example 1. Attenuation and phase difference for $z z$ coupling using short-spacing.

well within the most resistive layer. Then, as we move away from the bed boundary, we observe a decrease in the absolute value of the geosignal's attenuation. Coaxial measurements further confirm these observations. Figure 17 shows similar measurements for the second trajectory $T_{2}$. As in the previous case, we observe an increase in the absolute value of the geosignal's attenuation as we approach a more conductive layer. Considering a variable resistivity profile for the OWT zone helps us to navigate through the OWT zone and maximize the reservoir exploitation.

\section{Conclusions}

In oil reservoir, it is customary to consider and OWC as a planar interface in between the oil and water satu- rated rocks. However, in the real life models, due to the presence of capillary actions, the interface between oil and water saturated rocks takes the form of an OWT zone. The oil saturation, and consequently resistivity value in an OWT zone decreases almost exponentially as a function of depth. Therefore, in order to simulate the real life model problems, we need to be capable of modeling layers with arbitrary resistivity profiles.

Our proposed numerical $1.5 \mathrm{D}$ solver can consider layers with arbitrary resistivity distributions, which allows us to naturally simulate a reservoir which contains an OWT zone. The aforementioned physical consideration helps us to perform more realistic simulations and thus more accurately navigate through the reservoir, which may maximize its production. 


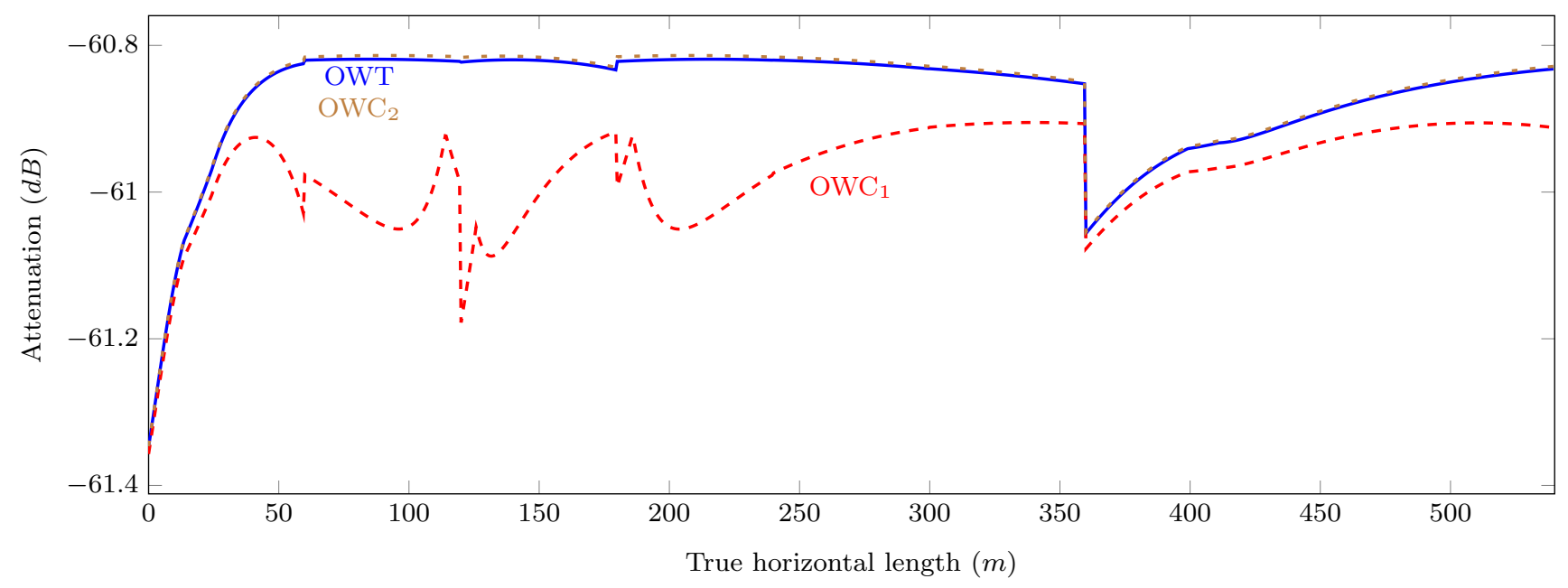

(a) Short spacing

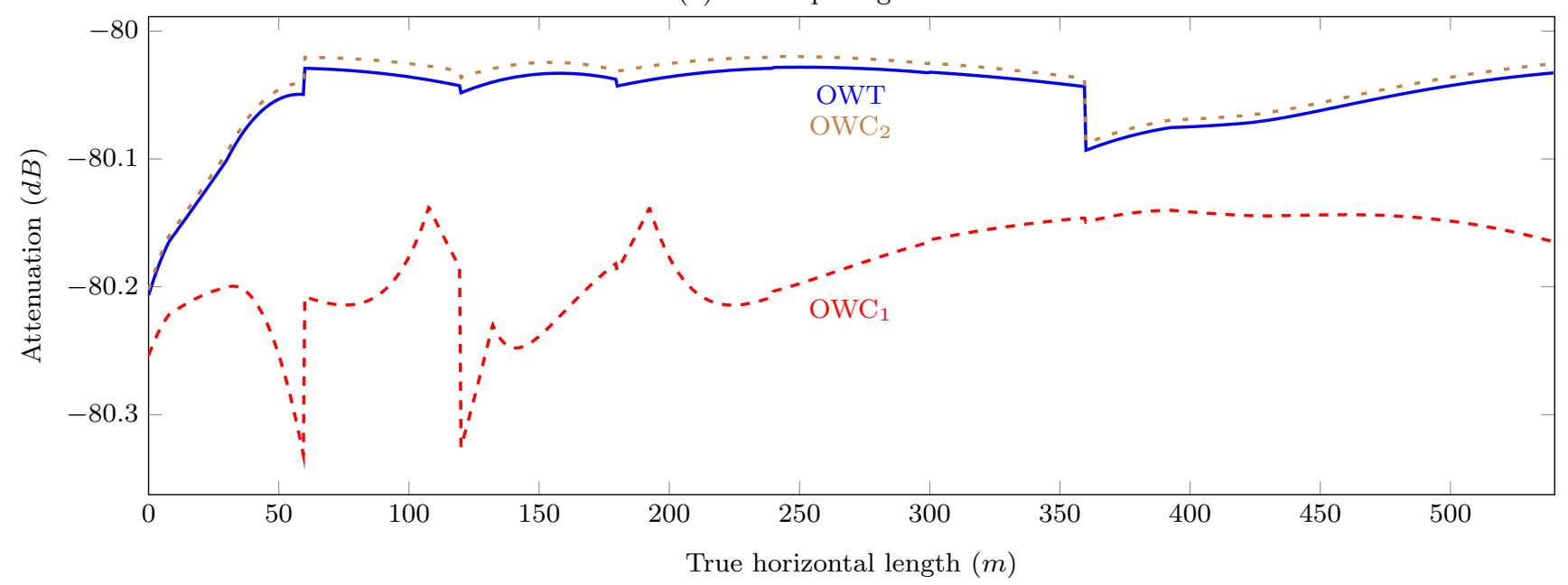

(b) Long spacing

Fig. 8: Example 1. Attenuation of $z z$ coupling using short and long spacings.

We demonstrated that considering and OWC model may lead to an extensive error in the inversion and navigation. The aforementioned error may direct the instrument towards a partially water-saturated rock. Hence, significantly reducing the posterior production from the reservoir. Considering an OWT zone and an efficient method which can simulate it is vital to maximize the reservoir production.

As a future work, we shall employ the $1.5 \mathrm{D}$ solver as a primary field for $2.5 \mathrm{D}$ and $3 \mathrm{D}$ simulations to build accurate and fast numerical simulators. Moreover, it will be possible to combine OWT zones with the presence of geological faults.

Acknowledgements Mostafa Shahriari has been supported by the Austrian Ministry for Transport, Innovation and Tech- nology, the Federal Ministry for Digital and Economic Affairs, and the Province of Upper Austria in the frame of the COMET center SCCH.

David Pardo has received funding from the European Union's Horizon 2020 research and innovation programme under the Marie Sklodowska-Curie grant agreement No 777778 (MATHROCKS), the POCTEFA H2020 Project PIXIL

(EFA362/19), the Project of the Spanish Ministry of Economy and Competitiveness with reference MTM2016-76329-R (AEI/FEDER, EU), the BCAM "Severo Ochoa" accreditation of excellence (SEV-2017-0718), and the Basque Government through the BERC 2018-2021 program, the two Elkartek projects ArgIA (KK-2019-00068) and MATHEO (KK2019-00085), and the Consolidated Research Group MATHMODE (IT1294-19) given by the Department of Education. 


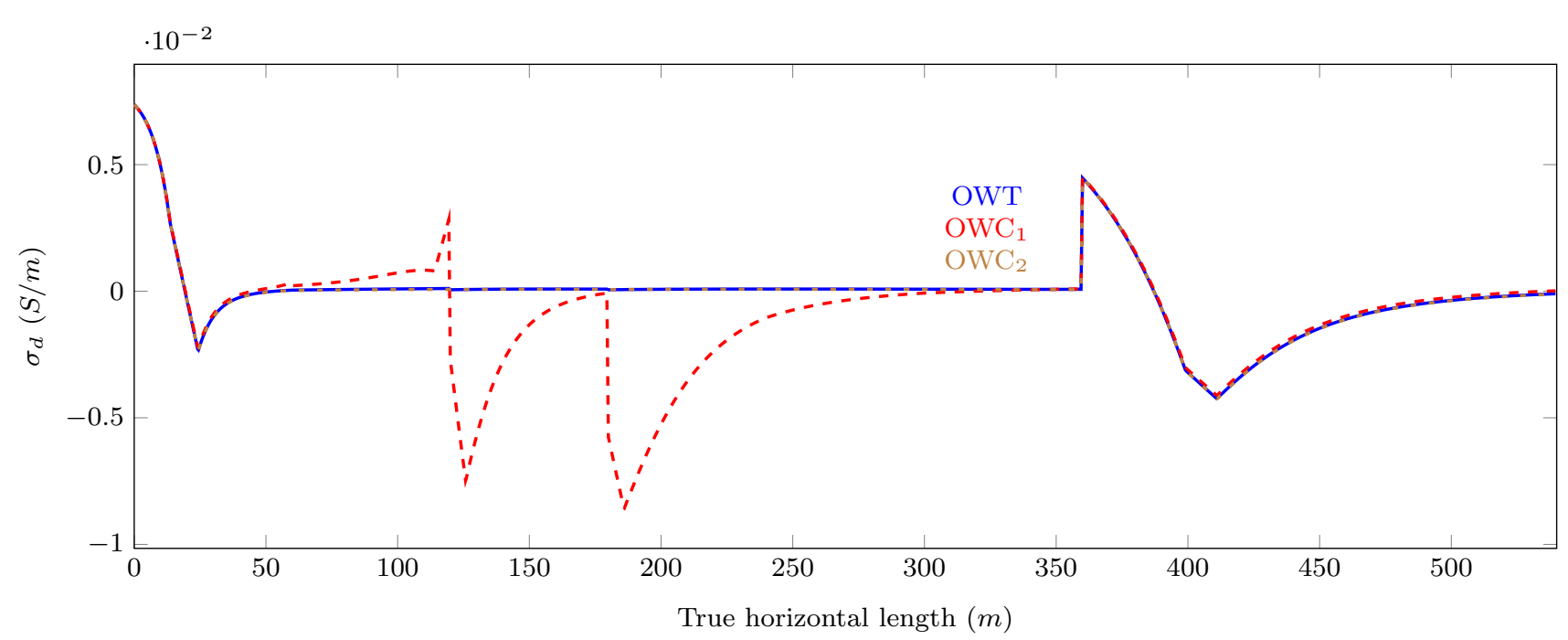

(a) Short spacing

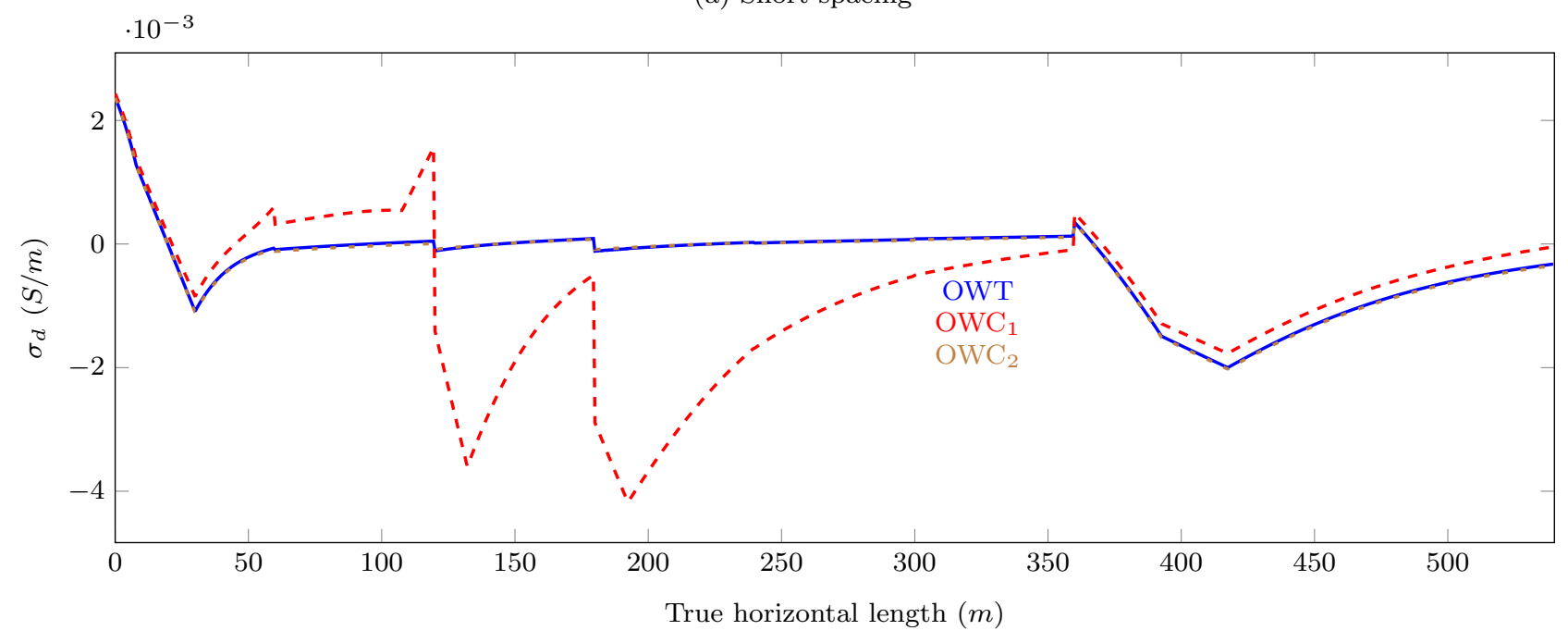

(b) Long spacing

Fig. 9: Example 1. Real part of symmetrized apparent conductivity using short and long spacings.

\section{References}

1. Bakr, S.A., Pardo, D., Mannseth, T.: Domain decomposition Fourier FE method for the simulation of 3D marine CSEM measurements. Journal of Computational Physics 255, 456-470 (2013)

2. Streich, R., Becken, M.: Sensitivity of controlledsource electromagnetic fields in planarly layered media. Geophysical Journal International 187, 705-728 (2011)

3. Constable, S., Srnka, L.J.: An introduction to marine controlled-source electromagnetic methods for hydrocarbon exploration. Geophysics 72 (2), WA3-WA12 (2007)

4. Pardo, D., Nam, M.J., Torres-Verdin, C., Hoversten, M.G., Garay, I.: Simulation of marine con- trolled source electromagnetic measurements using a parallel Fourier $h p$-finite element method. Computational Geosciences 15, 53-67 (2011)

5. Key, K.: 1D inversion of multicomponent, multifrequency marine CSEM data: Methodology and synthetic studies for resolving thin resistive layers. Geophysics 74 (2), F9-F20 (2009)

6. Martí, A.: The role of electrical anisotropy in magnetotelluric responses: from modelling and dimensionality analysis to inversion and interpretation. Surveys in Geophysics 35, 179-218 (2014)

7. Alvarez-Aramberri, J., Pardo, D.: Dimensionally adaptive $h p$-finite element simulation and inversion of 2D magnetotelluric measurements. Journal of Computational Science 18, 95-105 (2017) 


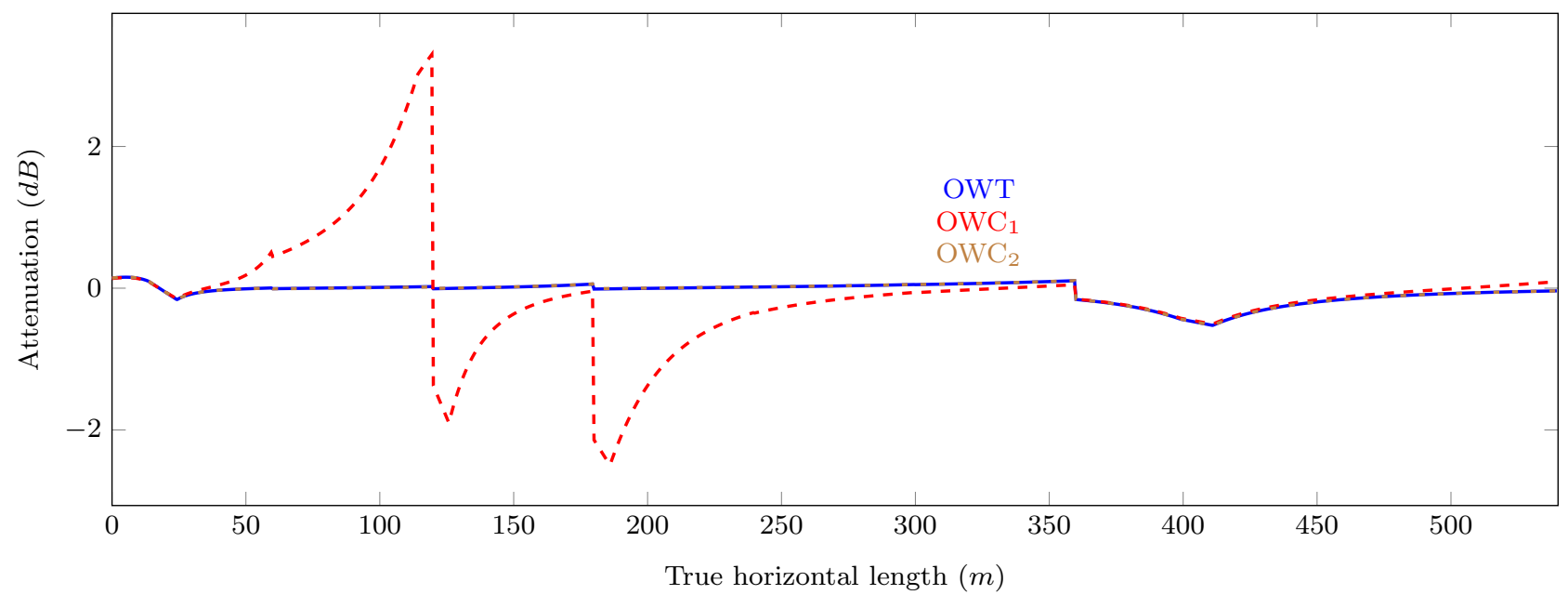

(a) Short spacing

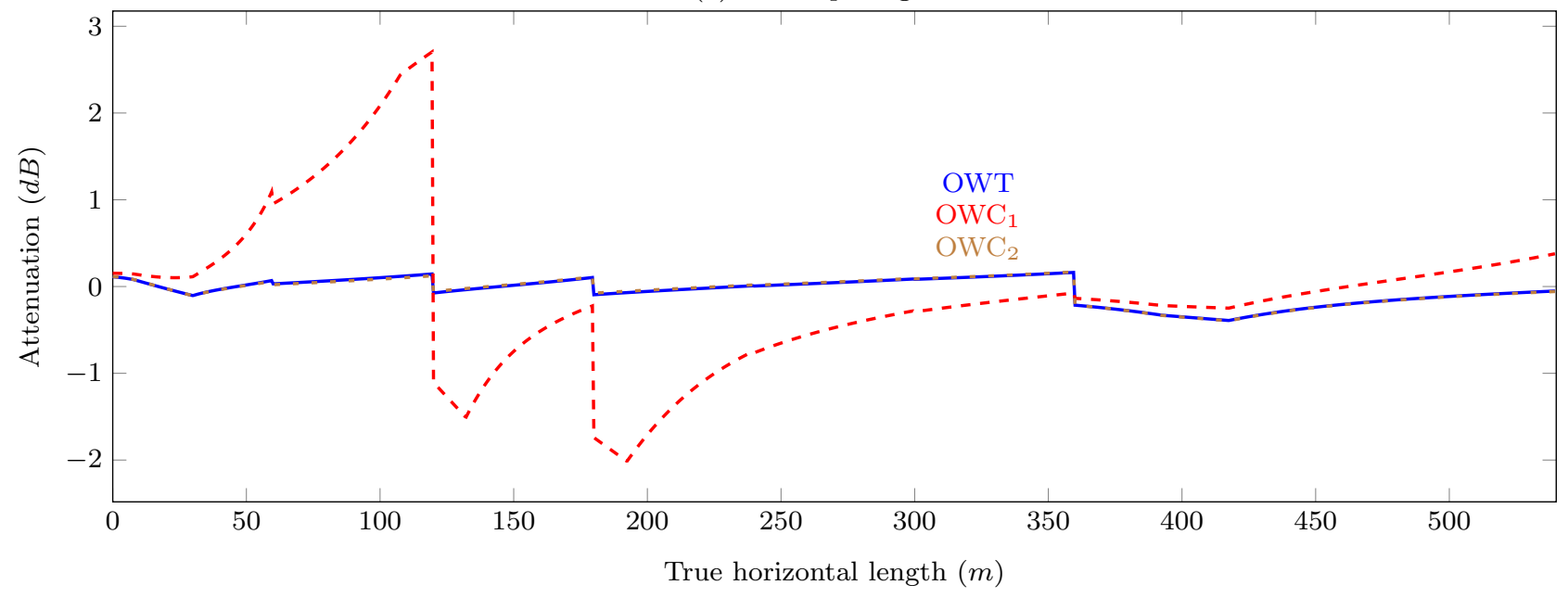

(b) Long spacing

Fig. 10: Example 1. Attenuation of geosignal using short and long spacings.

8. Pardo, D., Torres-Verdin, C.: Fast 1D inversion of logging-while-drilling resistivity measurements for the improved estimation of formation resistivity in high-angle and horizontal wells. Geophysics 80 (2), E111-E124 (2014)

9. Davydycheva, S., Wang, T.: A fast modelling method to solve Maxwell's equations in 1D layered biaxial anisotropic medium. Geophysics 76 (5), F293-F302 (2011)

10. Wang, G.L., Barber, T., Wu, P., Allen, D., Abubakar, A.: Triaxial induction tool response in dipping and crossbedded formations. Society of Exploration Geophysicists pp. 585-590 (2014)

11. Ijasana, O., Torres-Verdín, C., Preeg, W.E.: Inversion-based petrophysical interpretation of logging-while-drilling nuclear and resistivity measurements. Geophysics 78 (6), D473-D489 (2013)
12. Davydycheva, S., Homan, D., Minerbo, G.: Triaxial induction tool with electrode sleeve: FD modeling in 3D geometries. Journal of Applied Geophysics 67, 98-108 (2004)

13. Seydoux, J., Legendre, E., Mirto, E., Dupuis, C., Denichou, J.M., Bennett, N., Kutiev, G., Kuchenbecker, M., Morriss, C., Yang, L.: Full 3D deep directional resistivity measurements optimize well placement and provide reservoir-scale imaging while drilling. Society of Petrophysicists and WellLog Analysts pp. 1-14 (2014)

14. Bell, C., Hampson, J., Eadsforth, P., Chemali, R., Helgesen, T., Meyer, H., Peveto, C., Poppitt, A., Randall, R., Signorelli, J., Wang, T.: Navigating and imaging in complex geology with azimuthal propagation resistivity while drilling. Society of Petrophysicists and Well-Log Analysts pp. 1-14 (2006) 


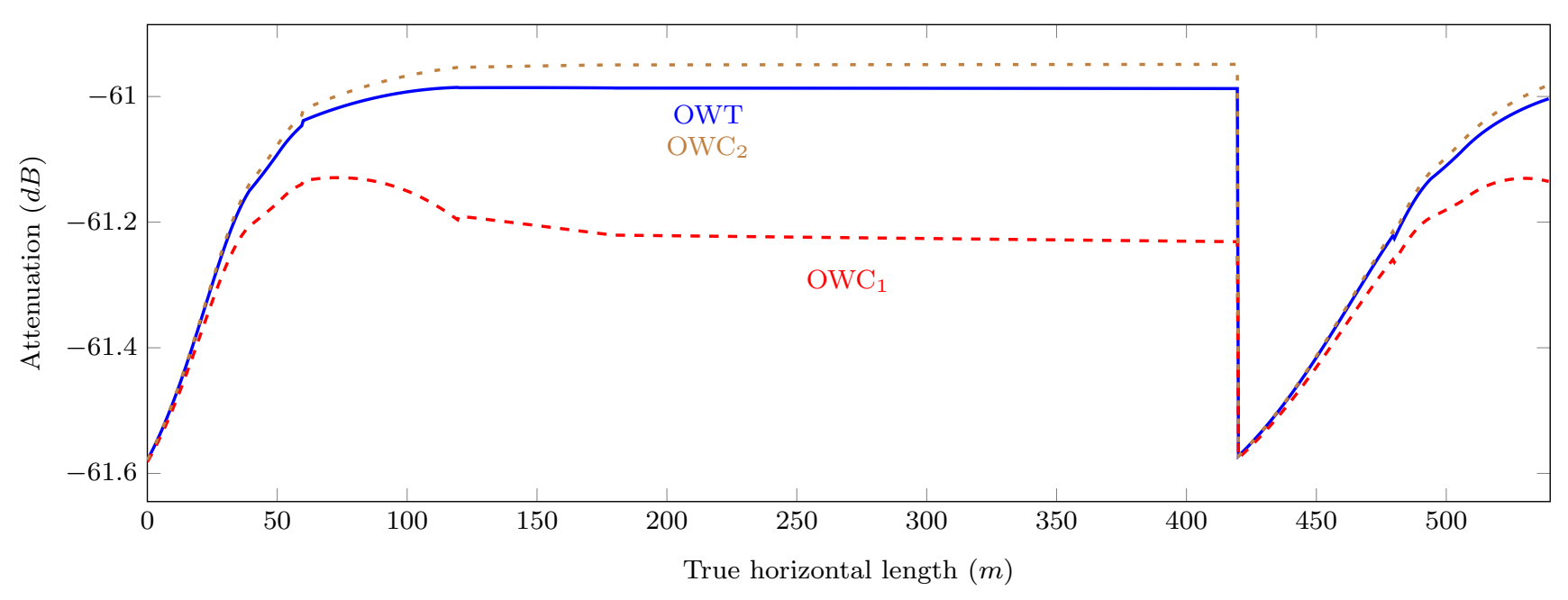

(a) Short spacing

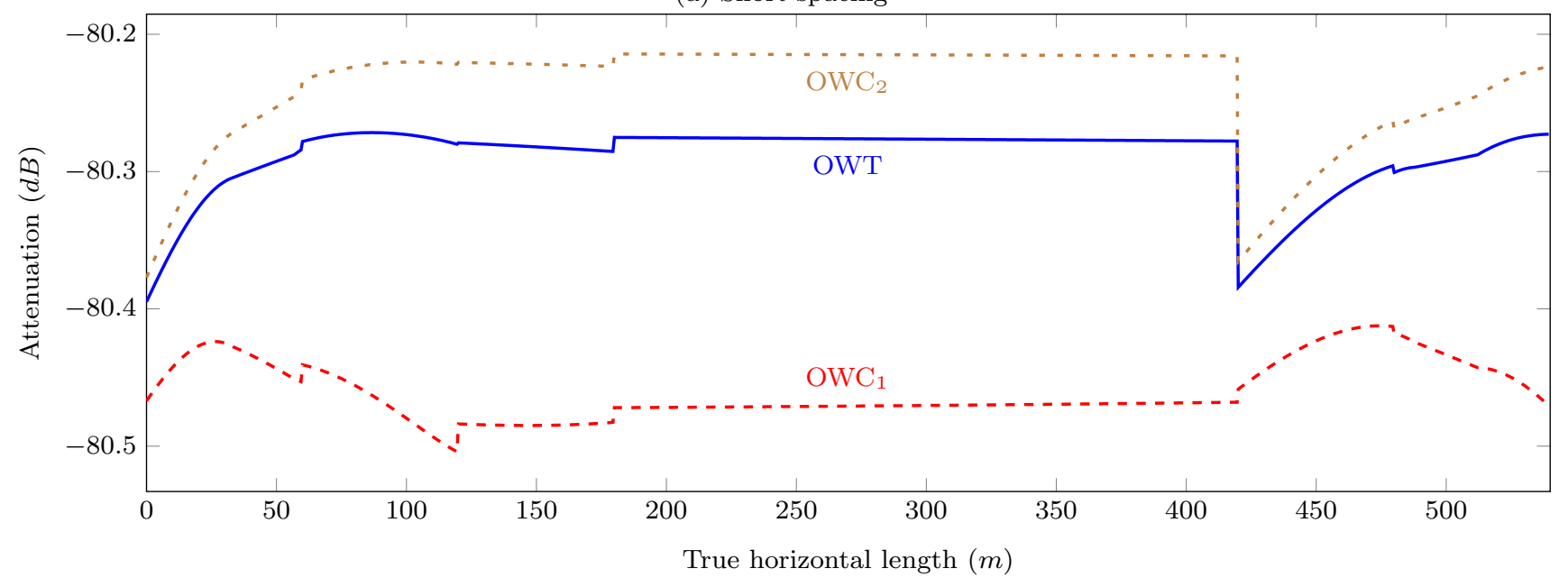

(b) Long spacing

Fig. 11: Example 2. Attenuation of $z z$ coupling using short and long spacings for trajectory $T_{1}$.

15. Bittar, M., Klein, J., Beste, R., Hu, G., Wu, M., Pitcher, J., Golla, C., Althoff, G., Sitka, M., Minosyam, V., Paulk, M.: A new azimuthal deepreading resistivity tool for geosteering and advanced formation evaluation. Society of Petrophysicists and Well-Log Analysts pp. 1-10 (2009)

16. Chemali, R., Bittar, M., Hveding, F., Wu, M., Dautel, M.: Improved geosteering by integrating in real time images from multiple depths of investigation and inversion of azimuthal resistivity signals. Society of Petrophysicists and Well-Log Analysts pp. 1-7 (2010)

17. Davydycheva, S.: Separation of azimuthal effects for new-generation resistivity logging tools - part 1. Geophysics 75(1), E31-E40 (2010)
18. Davydycheva, S.: Separation of azimuthal effects for new-generation resistivity logging tools - part 2. Geophysics 76(3), F185-F202 (2011)

19. Shahriari, M., Rojas, S., Pardo, D., RodríguezRozas, A., Bakr, S.A., Calo, V.M., Muga, I.: A numerical 1.5D method for the rapid simulation of geophysical resistivity measurements. Geosciences 8(6), 1-28 (2018)

20. Omeragic, D., Habashy, T., Chen, Y.H., Polyakov, V., Kuo, C., Altman, R., Hupp, D., Maeso, C.: Reservoir characterization and well placement in complex scenarios using LWD directional EM measurements. Petrophysics 50, 396-415 (2009)

21. Al-Musharfi, N.M., Bansal, R., Ahmed, M.S., Kanj, M.Y., Morys, M., Conrad, C., Parker, T.J.: Real time reservoir characterization and geosteering us- 


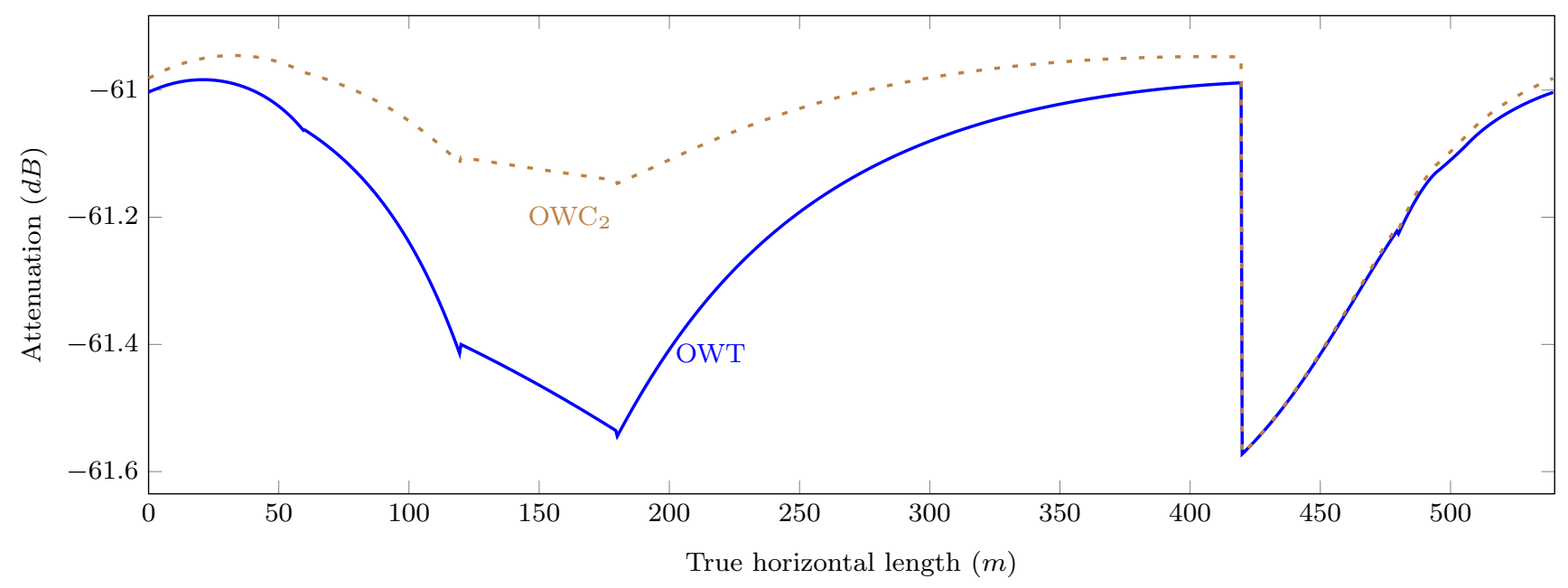

(a) Attenuation

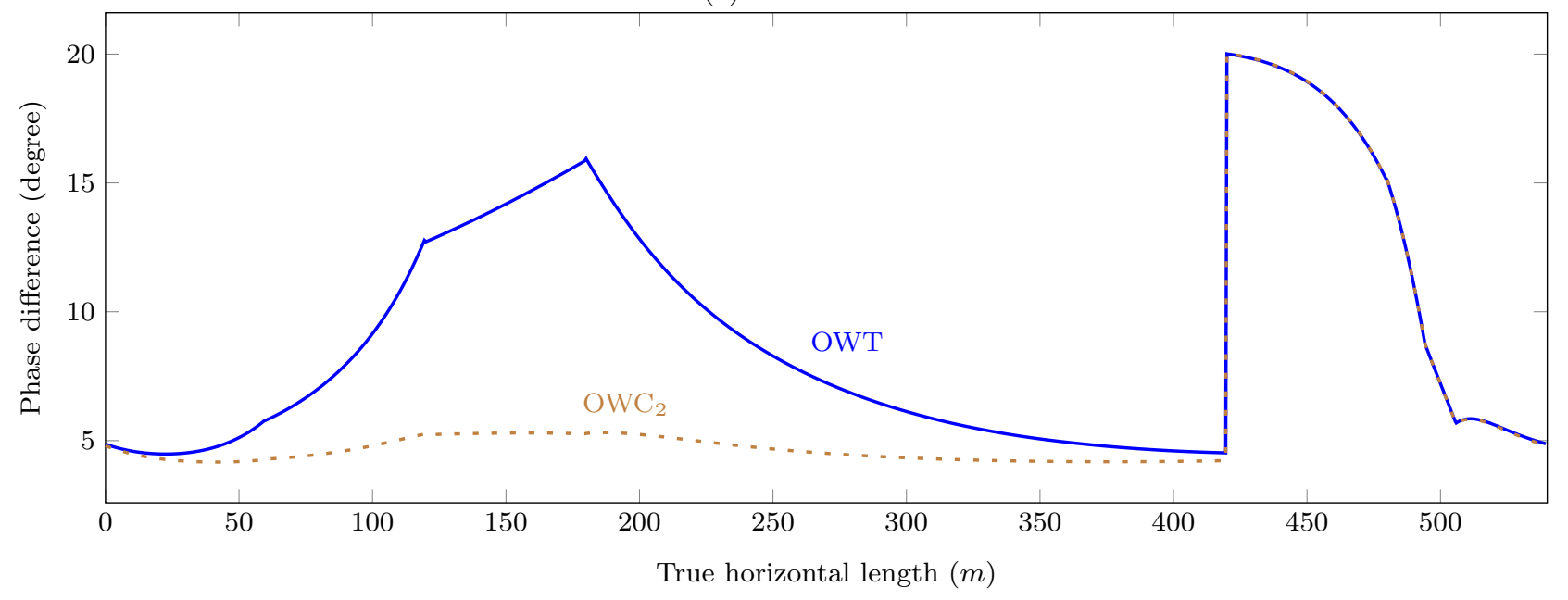

(b) Phase difference

Fig. 12: Example 2. Attenuation and phase difference for $z z$ coupling using short spacing for trajectory $T_{2}$.

ing advanced high-resolution LWD resistivity imaging. Society of Petroleum Engineers pp. 1-11 (2010)

22. Beer, R., Dias, L.C.T., da Cunha, A.M.V., Coutinho, M.R., Schmitt, G.H., Seydoux, J., Guedes, A.B.F.: Geosteering and/or reservoir characterization the prowess of new-generation LWD tools. Society of Petrophysicists and Well-Log Analysts pp. 1-14 (2010)

23. Zhang, C., Wu, Q., Wang, X., Lu, N.: Application of rotary geosteering drilling in deep and thin reservoirs of tarim basin, NW China. Petroleum Exploration and Development 40(6), $801-805$ (2013)

24. Kok, J.C.L., DeJarnett, J., Geary, D., Vauter, E.: Successful geosteering in low resistivity contrast reservoirs of the permian basin. Society of Petroleum Engineers pp. $1-4$ (2011)
25. Loseth, L.O., Ursin, B.: Electromagnetic fields in planarly layered anisotropic media. Geophysical Journal International 170, 44-F80 (2007)

26. Rojas, S., Muga, I., Pardo, D.: A quadrature-free method for simulation and inversion of $1.5 \mathrm{D}$ direct current (DC) borehole measurements. Computational Geosciences 20 (6), 1301-1318 (2016)

27. Lian, P., Tan, X.Q., Ma, C.Y., Feng, R.Q., Gao, H.M.: Saturation modeling in a carbonate reservoir using capillary pressure based saturation height function: a case study of the svk reservoir in the y field. Journal of Petroleum Exploration and Production Technology 6 (1), 73-84 (2016)

28. Ghasemi, M., Yang, Y., Gildin, E., Efendiev, Y., Calo, V.M.: Fast multiscale reservoir simulations using pod-deim model reduction. Society of Petroleum Engineers pp. 1-18 (2015) 


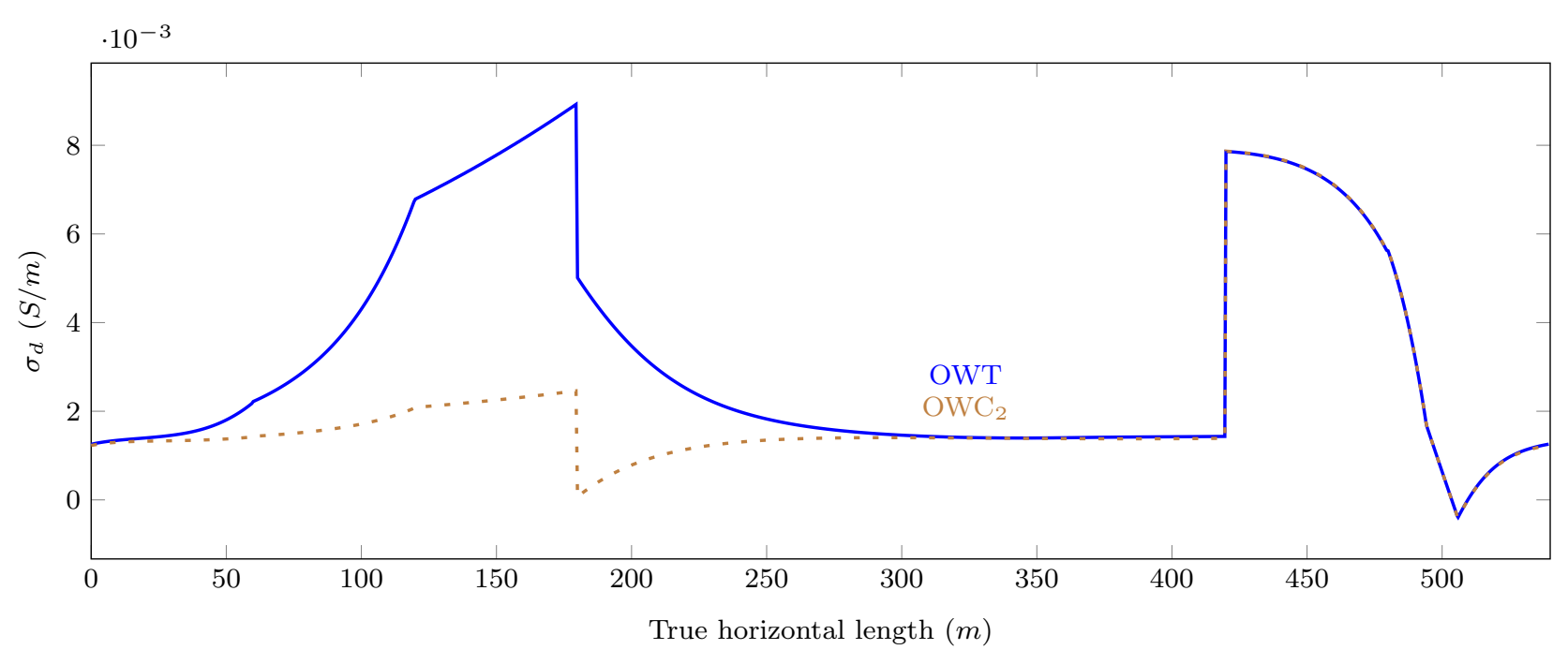

(a) Short spacing

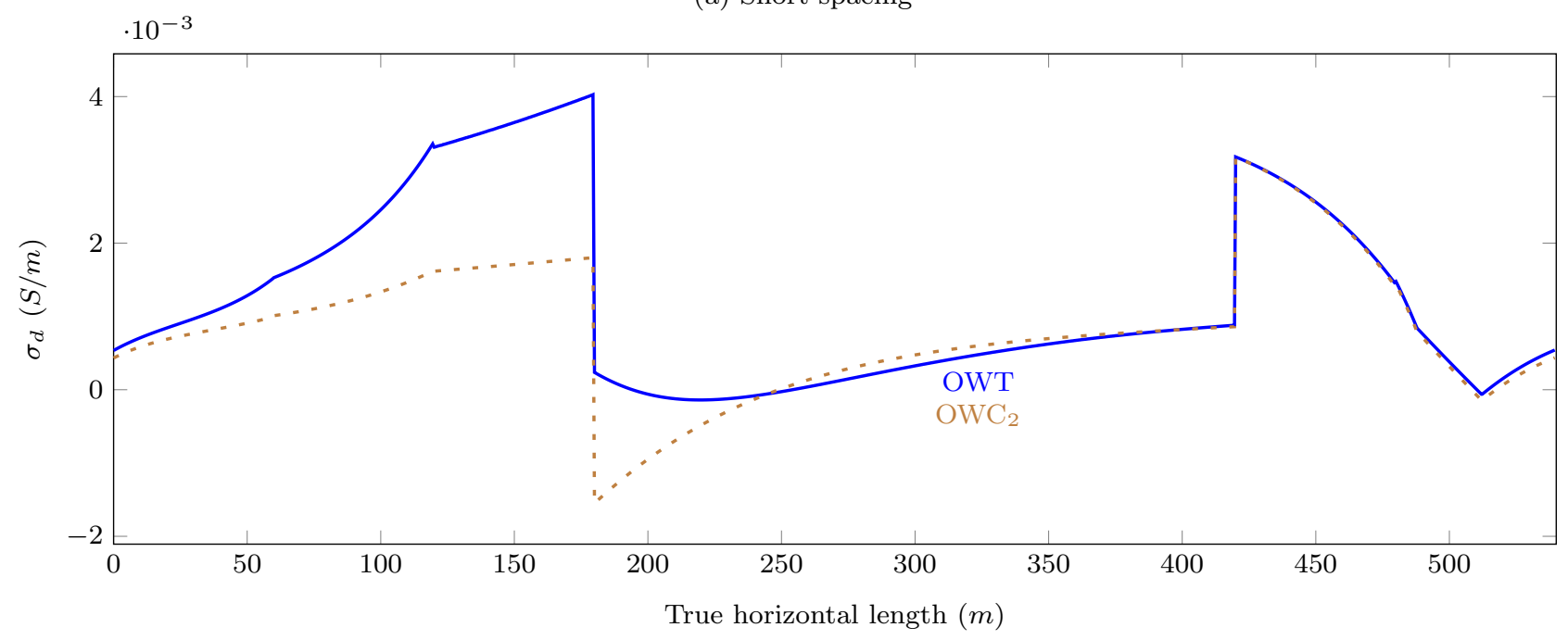

(b) Long spacing

Fig. 13: Example 2. Real part of symmetrized apparent conductivity using short and long spacings spacing for trajectory $T_{2}$.

29. Malekzadeh, F.A., Dusseault, M.B.: A solution for the transition zone isosats in two-phase primary drainage in the presence of gravity. Computational Geosciences 17 (5), 757-771 (2013)

30. Bhattacharya, S., Byrnes, A., Gerlach, P.: Costeffective integration of geologic and petrophysical characterization with material balance and decline curve analysis to develop a 3D reservoir model for pc-based reservoir simulation to design a waterflood in a mature Mississippian carbonate field with limited log data. Tech. Rep. no. 2003-31, Kansas Geological Survey, Open-file Report (2003)

31. Glover, P.W.J.: Archie's law - a reappraisal. Solid Earth 7(4), 1157-1169 (2016)
32. Nédélec, J.C.: Acoustic and electromagnetic equations, Applied Mathematical Sciences, vol. 144. Springer-Verlag, New York (2001). Integral representations for harmonic problems

33. Habashy, T., Anderson, B.: Reconciling differences in depth of investigation between $2-\mathrm{MHz}$ phase shift and attenuation resistivity measurements. Paper E presented at the 1991 SPWLA Annual Logging Symposium, Midland, Texas, 16-19 June (1991)

34. Desbrandes, R., Clayton, R.: Chapter 9 measurement while drilling. Developments in Petroleum Science 38, 251 - 279 (1994)

35. Seifert, D.J., Dossary, S.A., Chemali, R.E., Bittar, M.S., Lotfy, A.A., Pitcher, J.L., Bayrakdar, M.A.: Deep electrical images, geosignal, and real-time in- 


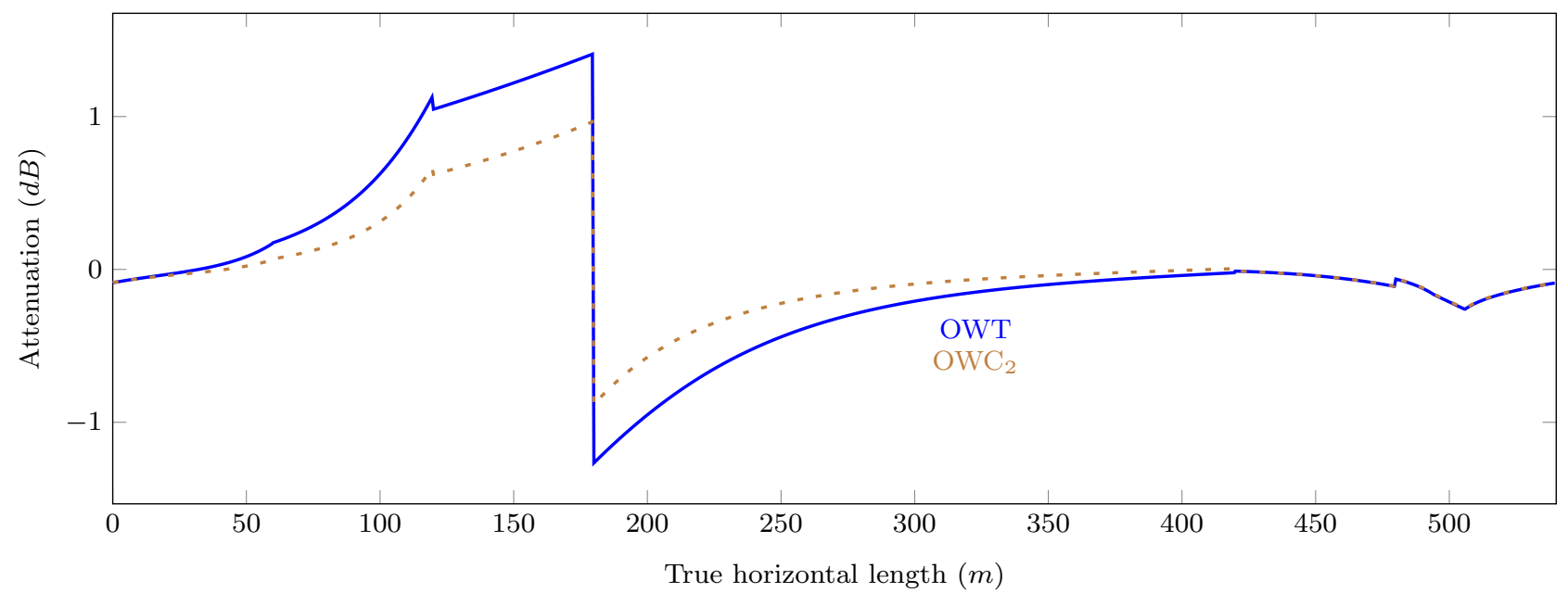

(a) Short spacing

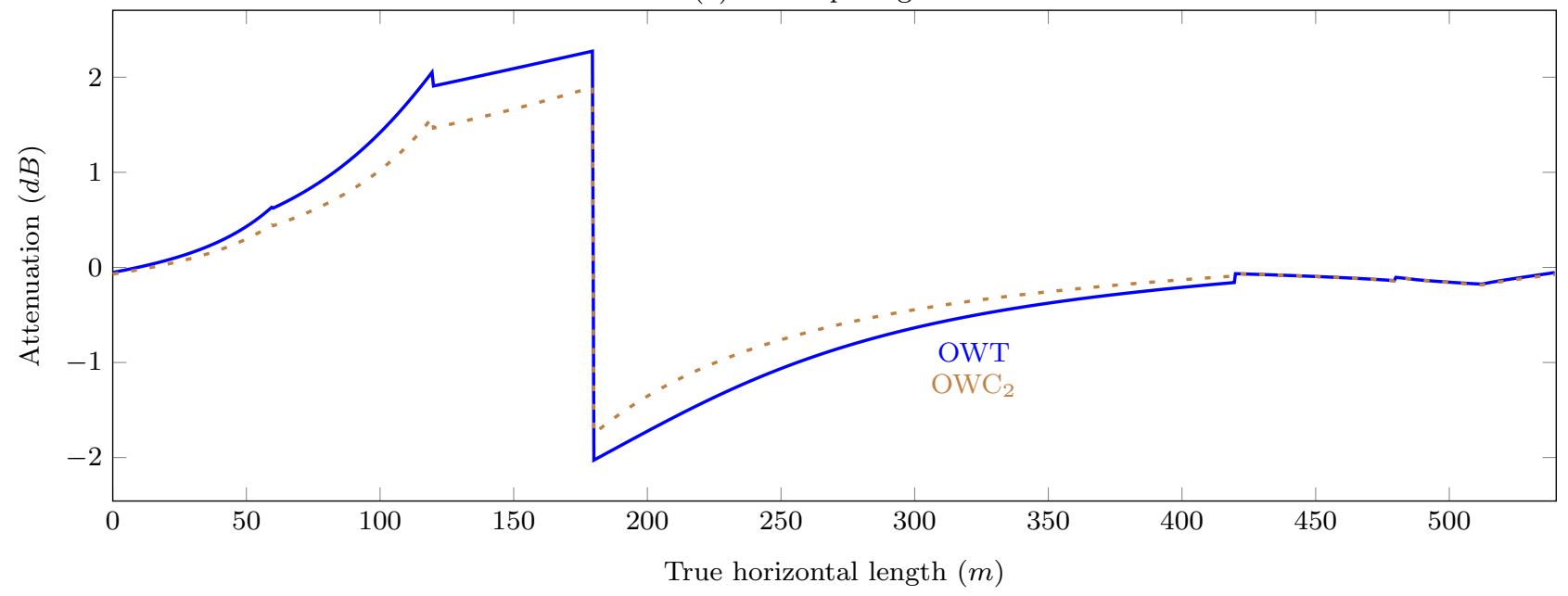

(b) Long spacing

Fig. 14: Example 2. Attenuation of the geosignal using short and long spacings for trajectory $T_{2}$.

version help guide steering decisions. Society of

Petroleum Engineers pp. 1-9 (2009) 


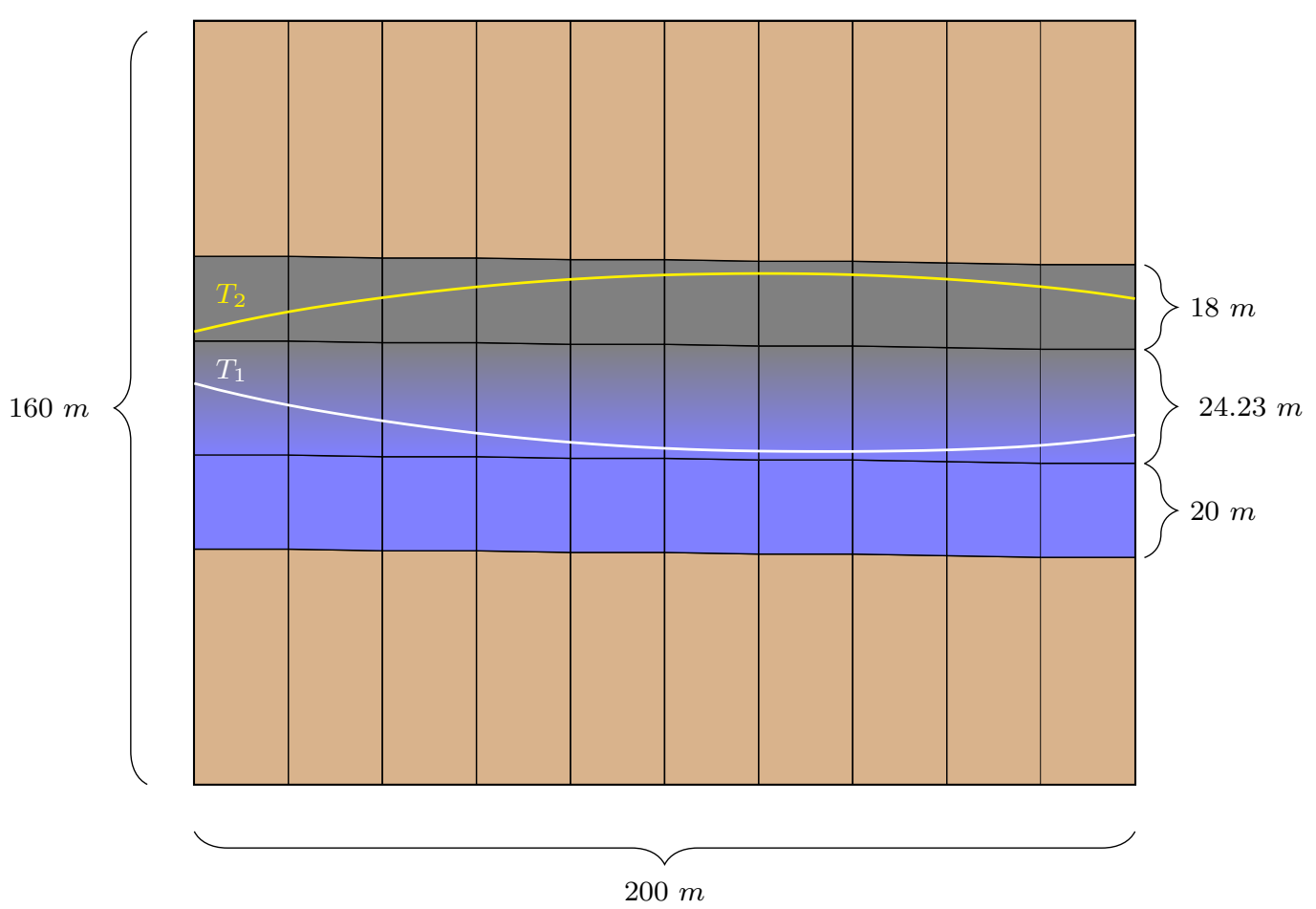

Fig. 15: Example 3. $T_{1}$ and $T_{2}$ describe the logging trajectories. Horizontal length of each vertical column is $20 \mathrm{~m}$. 


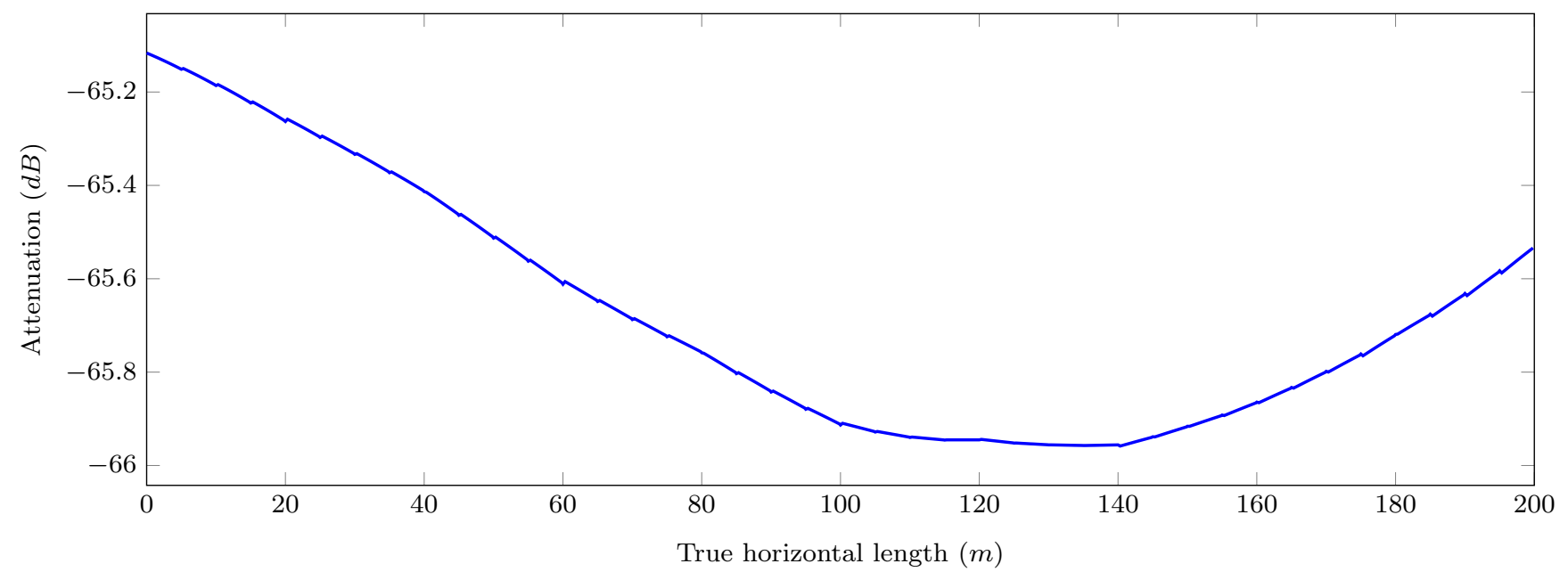

(a) Co-axial measurement

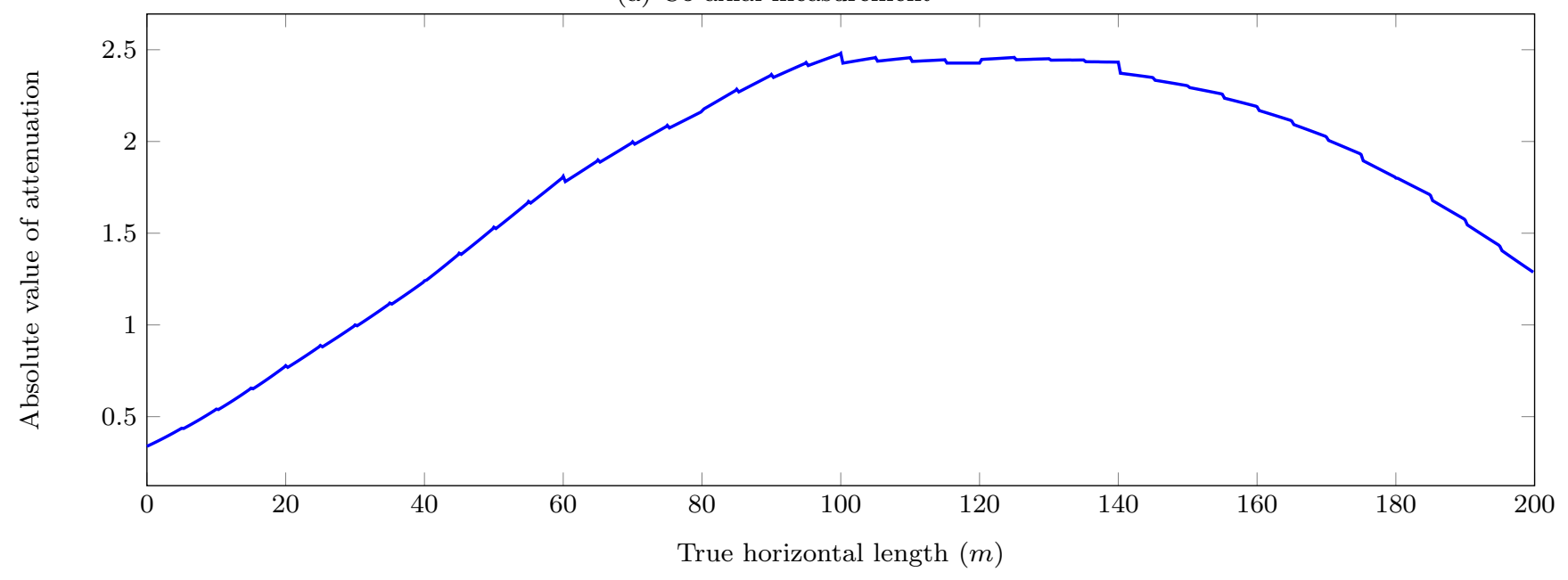

(b) Geosignal

Fig. 16: Example 3. Logs associated with the trajectory $T_{1}$. 


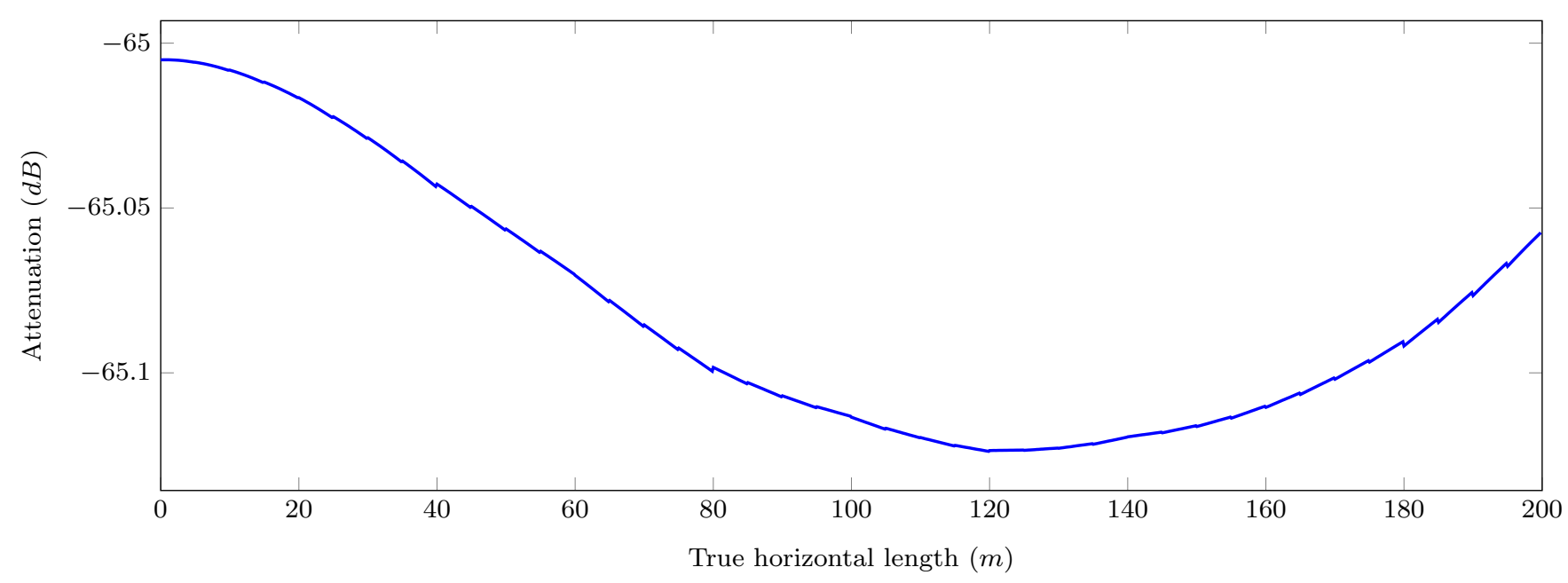

(a) Co-axial measurement

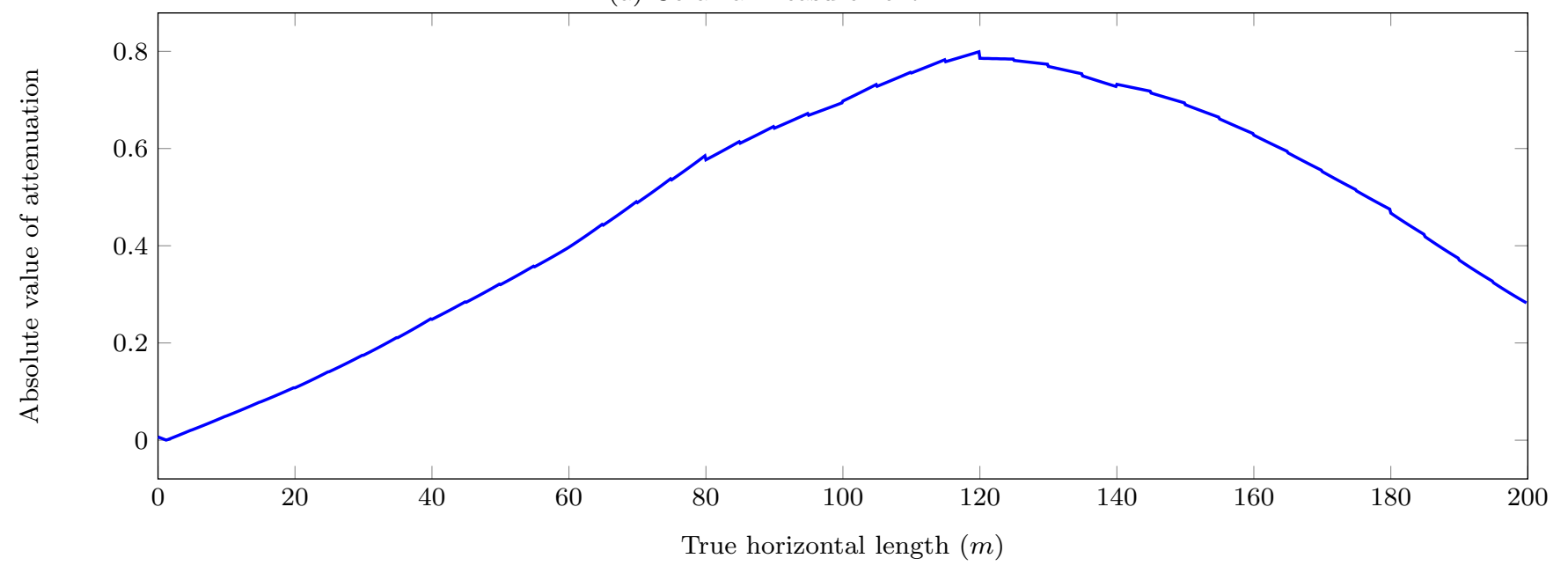

(b) Geosignal

Fig. 17: Example 3. Logs associated with the trajectory $T_{2}$. 